\title{
ON SEMISIMPLE HOPF ALGEBRAS OF DIMENSION $2^{m}$, II.
}

\author{
YEVGENIA KASHINA
}

\begin{abstract}
In this paper we classify, up to equivalence, all semisimple nontrivial Hopf algebras of dimension $2^{2 n+1}$ for $n \geq 2$ over an algebraically closed field of characteristic 0 with the group of group-like elements isomorphic to $\mathbb{Z}_{2^{n}} \times \mathbb{Z}_{2^{n}}$. Moreover we classify all such nonisomorphic Hopf algebras of dimension 32 and show that they are not twist-equivalent to each other. More generally, given an abelian group of order $2^{m-1}$ we give an upper bound for the number of nonisomorphic nontrivial Hopf algebras of dimension $2^{m}$ which have this group as their group of group-like elements.
\end{abstract}

\section{INTRODUCTION}

An important problem in the theory of semisimple Hopf algebras is to construct examples satisfying certain properties and in the second step classify all Hopf algebras with these properties. Several such classification results were obtained in recent years, in particular in the series of papers by Masuoka (see, for example, [Ma1, Ma2, [Ma3], [Ma4]) and by Natale (see, for example [Na1], [Na2], [Na3], [Na4, [Na5], [Na6]) as well as in a recent preprint $[\mathrm{Kr}$ by Krop.

In this paper we continue the project of classifying semisimple Hopf algebras of dimension $2^{m}$ over an algebraically closed field of characteristic 0 started in the papers [K1] and [K2]. In [K1] we have shown that there are exactly 16 nontrivial (that is, noncommutative and noncocommutative) semisimple Hopf algebras of dimension 16. In [K2 we have classified all nontrivial semisimple Hopf algebras of dimension $2^{n+1}$ for $n \geq 4$ with the group of group-like elements isomorphic to $\mathbb{Z}_{2^{n-1}} \times \mathbb{Z}_{2}$.

We now classify, up to equivalence, all semisimple nontrivial Hopf algebras of dimension $2^{2 n+1}$ for $n \geq 2$ over an algebraically closed field of characteristic 0 with the group of group-like elements isomorphic to $\mathbb{Z}_{2^{n}} \times \mathbb{Z}_{2^{n}}$. We then give the complete list of nonisomorphic nontrivial Hopf algebras of dimension 32 with the group of group-like elements isomorphic to $\mathbb{Z}_{4} \times \mathbb{Z}_{4}$. We also show that these Hopf algebras are not twist-equivalent to each other. The above-mentioned Hopf algebras are all obtained as abelian extensions of $k L$ by $k^{G}$ where $L \cong \mathbb{Z}_{2}$ and $G \cong \mathbb{Z}_{2^{n}} \times \mathbb{Z}_{2^{n}}$. Our ability to describe all such extensions heavily depends on the structure of $G$, and in particular on the number of order 2 automorphisms of $G$ and on the structure of the Schur multiplier $M(G)$. 
The paper is organized as follows: In Section 2 we gather the necessary preliminaries, in particular we discuss the cohomological description of abelian extensions based on works of Hofstetter, Masuoka, and Mastnak.

In Section 3 we consider a group homomorphism $\Psi$ from the group of equivalence classes of abelian extensions of $k L$ by $k^{G}, \mathrm{H}^{2}\left(k L, k^{G}\right)$, to the Schur multiplier $M(G)$ (for a fixed action of $L \cong \mathbb{Z}_{2}$ on an arbitrary group $G$ of order $2^{m-1}$ ). We show that its kernel is isomorphic to $\mathrm{H}^{2}(L, \hat{G})$ and that its image lies inside certain subgroup $M(G)^{-}$. This implies that $\left|\mathrm{H}^{2}\left(k L, k^{G}\right)\right| \leq\left|\mathrm{H}^{2}(L, \hat{G})\right| \cdot\left|M(G)^{-}\right|$. Moreover we conclude that there are at most $N(|\mathbf{G}| \cdot|M(\mathbf{G})| / 2-1)$ nonisomorphic nontrivial Hopf algebras of dimension $2^{m}$ with group of group-like elements isomorphic to an abelian group $\mathbf{G}$ of order $2^{m-1}$, where $N$ is the number of distinct conjugacy classes of elements of order 2 in $\operatorname{Aut}(\mathbf{G})$.

In Section 4 we describe, up to equivalence, all possible non-commutative semisimple Hopf algebras of dimension $2^{2 n+1}$ with group of group-like elements isomorphic to $\mathbb{Z}_{2^{n}} \times \mathbb{Z}_{2^{n}}$ for $n \geq 2$. Every Hopf algebra under consideration fits into an abelian extension of $k L$ by $k^{G}$. In Theorem 4.3 we show that for a fixed action of $L$ on $G$

$$
\mathrm{H}^{2}\left(k L, k^{G}\right) \cong \operatorname{Ker} \Psi \times \operatorname{Im} \Psi \cong \mathrm{H}^{2}(L, \hat{G}) \times M(G)^{-} .
$$

In Section 5 we describe all semisimple Hopf algebras of dimension 32 with $\mathbf{G}(H) \cong$ $\mathbb{Z}_{4} \times \mathbb{Z}_{4}$ and therefore prove the following theorem:

THEOREM 5.7. There are exactly 16 nontrivial non-isomorphic semisimple Hopf algebras of dimension 32 with group of group-like elements isomorphic to $\mathbb{Z}_{4} \times \mathbb{Z}_{4}$. Moreover, all of them can be distinguished by categorical invariants, such as their Grothendieck ring structure or their (higher) Frobenius-Schur indicators. Therefore, the corresponding representation categories are also different and these Hopf algebras are not twist-equivalent to each other.

The data required to distinguish non-isomorphic Hopf algebras is obtained in Section 5 and is gathered in Table 1. $N_{m, j}$ stands for the number of 2-dimensional irreducible representations of $H$ with $m$-th Frobenius-Schur indicator equal to $j$. Note that by [LM, Theorem 3.1], $N_{2, j}=0$ unless $j=-1,0,1$. For non-abelian groups of order 16 we use the notation from [K1, Section 4]:

$$
\begin{aligned}
& G_{1}=\left\langle a, b \mid a^{8}=b^{2}=1, b a b=a^{5}\right\rangle \\
& G_{6}=\left\langle a, b, c \mid a^{4}=b^{2}=c^{2}=1, b a b=a c\right\rangle
\end{aligned}
$$


Table 1

\begin{tabular}{|c|c|c|c|c|c|c|}
\hline & $G \cap Z(H)$ & $\mathbf{G}\left(H^{*}\right)$ & $N_{2,0}$ & $N_{2,1}$ & $N_{2,-1}$ & Notes \\
\hline $\begin{array}{l}H\left(\rightarrow_{1}, \alpha_{1,2}^{2}\right) \\
H\left(\rightarrow_{1}, \alpha_{1,1}^{2} \alpha_{1,2}^{2} \alpha_{2,2}^{2}\right)\end{array}$ & $\mathbb{Z}_{2} \times \mathbb{Z}_{2}$ & $\left(\mathbb{Z}_{2}\right)^{3}$ & 0 & $\begin{array}{l}4 \\
0\end{array}$ & $\begin{array}{l}2 \\
6 \\
\end{array}$ & \\
\hline $\begin{array}{l}H\left(\overrightarrow{ }_{2}, \alpha_{1,2}\right) \\
H\left(\overrightarrow{ }_{2}, \alpha_{1,2}^{2}\right) \\
H\left(\overrightarrow{ }_{2}, \alpha_{1,2}^{2} \alpha_{2,2}^{2}\right) \\
H\left(\overrightarrow{ }_{2}, \beta_{1}, \alpha_{1,2}\right) \\
H\left(\overrightarrow{ }_{2}, \beta_{1}, \alpha_{1,2} \alpha_{2,2}^{2}\right) \\
H\left(\overrightarrow{ }_{2}, \beta_{1}, \alpha_{1,2}^{2}\right)\end{array}$ & $\mathbb{Z}_{2} \times \mathbb{Z}_{4}$ & $\begin{array}{l}G_{6} \\
\left(\mathbb{Z}_{2}\right)^{2} \times \mathbb{Z}_{4} \\
\left(\mathbb{Z}_{2}\right)^{2} \times \mathbb{Z}_{4} \\
G_{1} \\
G_{1} \\
\mathbb{Z}_{2} \times \mathbb{Z}_{8}\end{array}$ & 2 & $\begin{array}{l}1 \\
2 \\
0 \\
2 \\
0 \\
2\end{array}$ & $\begin{array}{l}1 \\
0 \\
2 \\
0 \\
2 \\
0\end{array}$ & \\
\hline $\begin{array}{l}H\left(\rightarrow_{3}, \alpha_{1,2}^{2}\right) \\
H\left(\rightarrow_{3}, \beta_{1}, \alpha_{1,2}^{2}\right)\end{array}$ & $\mathbb{Z}_{2} \times \mathbb{Z}_{4}$ & $\begin{array}{l}\left(\mathbb{Z}_{2}\right)^{2} \times \mathbb{Z}_{4} \\
\mathbb{Z}_{4} \times \mathbb{Z}_{4}\end{array}$ & 4 & 0 & 0 & \\
\hline $\begin{array}{l}H\left(\rightarrow_{4}, \alpha_{1,2}^{2}\right) \\
H\left(\rightarrow_{4}, \alpha_{1,2}^{2} \alpha_{2,2}^{2}\right)\end{array}$ & $\mathbb{Z}_{2} \times \mathbb{Z}_{2}$ & $\left(\mathbb{Z}_{2}\right)^{3}$ & 4 & $\begin{array}{l}2 \\
0 \\
\end{array}$ & $\begin{array}{l}0 \\
2 \\
\end{array}$ & \\
\hline $\begin{array}{l}H\left(\rightarrow_{5}, \alpha_{1,2}^{\prime}\right) \\
H\left(\rightarrow_{5},\left(\alpha_{1,2}^{\prime}\right)^{2}\right)\end{array}$ & $\mathbb{Z}_{4}$ & $\left(\mathbb{Z}_{2}\right)^{3}$ & 6 & 0 & 0 & $\begin{array}{l}N_{8,2}=2 \\
N_{8,2}=6\end{array}$ \\
\hline $\begin{array}{l}H\left(\rightarrow_{6}, \alpha_{1,1} \alpha_{1,2} \alpha_{2,2}\right) \\
H\left({ }_{6}, \alpha_{1,1}^{2} \alpha_{1,2}^{2} \alpha_{2,2}^{2}\right)\end{array}$ & $\mathbb{Z}_{2} \times \mathbb{Z}_{4}$ & $\mathbb{Z}_{2} \times \mathbb{Z}_{4}$ & 4 & 2 & 0 & $\begin{array}{l}N_{4,2}=0 \\
N_{4,2}=6\end{array}$ \\
\hline
\end{tabular}

\section{PRELiminaries.}

Throughout this paper we will assume that $k$ is an algebraically closed field of characteristic 0 and that $H$ is a semisimple Hopf algebra over $k$ with comultiplication $\Delta: H \longrightarrow H \otimes H$, defined via $h \mapsto \sum h_{1} \otimes h_{2}$, counit $\varepsilon: H \longrightarrow k$, and antipode $S$. We let $\mathbf{G}(H)$ denote the group of group-like elements of $H$. For a general reference on Hopf algebras, see [M].

Definition 2.1 ([Ma5, Definition 1.4]). Consider the sequence

$$
K \stackrel{\iota}{\hookrightarrow} H \stackrel{\pi}{\rightarrow} F
$$

of finite-dimensional Hopf algebras and Hopf algebra maps $\iota$ and $\pi$ where $\iota$ is injective and $\pi$ is surjective. This sequence is called an extension of $F$ by $K$ if $K=\left\{h \in H \mid \sum h_{1} \otimes \pi\left(h_{2}\right)=h \otimes 1_{F}\right\}$, where $K \subset H$ via $i$. We say that two extensions $K \hookrightarrow H \rightarrow F$ and $K \hookrightarrow H^{\prime} \rightarrow F$ are equivalent if there is a Hopf algebra isomorphism $f: H \rightarrow H^{\prime}$ which induces the identity maps on $K$ and $F$.

For a general reference on Hopf algebra extensions, see [H] and [Ma5].

Definition 2.2. Let $K$ and $F$ be Hopf algebras equipped with a weak action $\rightarrow$ : $F \otimes K \rightarrow K$, a weak coaction $\rho: F \rightarrow F \otimes K$, a cocycle $\sigma: F \otimes F \rightarrow K$ and a dual 
cocycle $\tau: F \rightarrow K \otimes K$. The bicrossed product $K \#_{\sigma}^{\tau} F$ is a Hopf algebra with $K \otimes F$ as an underlying vector space and the following multiplication and comultiplication:

$$
\begin{array}{r}
(x \# a)(y \# b)=\sum x\left(a_{1} \rightarrow y\right) \sigma\left(a_{2}, b_{1}\right) \# a_{3} b_{2} \\
\Delta(x \# a)=\sum x_{1} \tau_{i}\left(a_{1}\right) \#\left(a_{2}\right)^{0} \otimes x_{2} \tau^{i}\left(a_{1}\right)\left(a_{2}\right)^{1} \# a_{3}
\end{array}
$$

where

$$
\begin{array}{r}
\rho(a)=\sum a^{0} \otimes a^{1} \\
\tau(a)=\sum_{i} \tau_{i}(a) \otimes \tau^{i}(a)
\end{array}
$$

For the necessary conditions on $\rightarrow, \rho, \sigma$, and $\tau$ see [A, 3.1].

For the rest of the paper we assume that $K$ is commutative and $F$ is cocommutative. Then the extension (2.1) is called abelian; since $k$ is algebraically closed, it follows by $[\mathrm{M}, 2.3 .1]$ that $K \cong(k G)^{*}$ and $F \cong k L$, for two finite groups $G$ and $L$. Let $\left\{p_{g} \mid g \in G\right\}$ be the dual basis for $(k G)^{*}$.

By [Ma5, Proposition 1.5] any abelian extension of $F$ by $K$ is equivalent to an extension

$$
K \stackrel{\iota}{\hookrightarrow} K \#_{\sigma}^{\tau} F \stackrel{\pi}{\rightarrow} F
$$

where for $g \in G, a \in L$

$$
\begin{array}{r}
\iota\left(p_{g}\right)=p_{g} \# 1 \\
\pi\left(p_{g} \# a\right)=\delta_{1, g} a
\end{array}
$$

As in [KMM, page 891] the action $\rightarrow$ of $L$ on $K$ induces an action of $L$ on $K^{*}=k G$ via $(l \rightarrow f)(k):=f(S l \rightarrow k)$. Since $K$ is commutative and $k L$ is cocommutative, $K$ is a $k L$-module algebra, and thus $L$ acts as automorphisms of $K$. Thus $L$ permutes the orthogonal idempotents $\left\{p_{g}\right\}$; it follows that the action of $L$ on $k G$ is in fact an action of $L$ on $G$ itself, which we also denote by $\rightarrow$. Then the action of $L$ on $(k G)^{*}$ is given by

$$
x \rightarrow p_{g}=p_{x \rightarrow g} .
$$

Let us now fix the action $\rightarrow$ and coaction $\rho$. Equivalence classes of abelian extensions form the abelian group $\operatorname{Opext}(F, K) \cong \mathrm{H}^{2}(F, K)=\mathrm{Z}^{2}(F, K) / \mathrm{B}^{2}(F, K)$ (see [H, Section 3]), where $\mathrm{Z}^{2}(F, K)$ and $\mathrm{B}^{2}(F, K)$ are defined in [H , page 268]. Let us now assume that coaction $\rho$ is trivial. In this case more explicit description of $\mathrm{Z}^{2}(F, K)$ and $\mathrm{B}^{2}(F, K)$ can be found in [Mas2, Section 2]:

For $a, b \in L, g, h \in G$ write

$$
\begin{aligned}
\sigma(a, b) & =\sum_{g \in G} \sigma(g ; a, b) p_{g} \\
\tau(a) & =\sum_{g, h \in G} \tau(g, h ; a) p_{g} \otimes p_{h}
\end{aligned}
$$


where $\sigma(g ; a, b), \tau(g, h ; a) \in k^{\times}$, as in [Ma5, page 170]. Then the pair $(\sigma, \tau)$ lies in $\mathrm{Z}^{2}(F, K)$ if and only if for all $a, b, c \in L, g, h, k \in G$

$$
\begin{aligned}
\sigma\left(a^{-1} \rightarrow g ; b, c\right) \sigma(g ; a, b c) & =\sigma(g ; a, b) \sigma(g ; a b, c) \\
\sigma(1 ; a, b)=\sigma(g ; 1, b) & =\sigma(g ; a, 1)=1 \quad \text { normalization condition for } \sigma \\
\tau(g, h ; a) \tau(g h, k ; a) & =\tau(g, h k ; a) \tau(h, k ; a) \\
\tau(g, h ; 1)=\tau(1, h ; a) & =\tau(g, 1 ; a)=1 \quad \text { normalization condition for } \tau \\
\tau(g, h ; a b) \sigma(g h ; a, b) & =\tau(g, h ; a) \tau\left(a^{-1} \rightarrow g, a^{-1} \rightarrow h ; b\right) \sigma(g ; a, b) \sigma(h ; a, b)
\end{aligned}
$$

In addition, $\mathrm{B}^{2}(F, K)$ consists of pairs $(\sigma, \tau) \in \mathrm{Z}^{2}\left(k L, k^{G}\right)$ such that there exists $\gamma: L \rightarrow K^{\times}$defined via

$$
\gamma(a)=\sum_{g \in G} \gamma(g ; a) p_{g} \quad \text { for } a \in L
$$

satisfying

$$
\begin{aligned}
\gamma(g ; 1) & =\gamma(1 ; g)=1 \\
\sigma(g ; a, b) & =\gamma(g ; a) \gamma\left(a^{-1} \rightarrow g ; b\right) \gamma(g ; a b)^{-1} \\
\tau(g, h ; a) & =\gamma(g ; a)^{-1} \gamma(h ; a)^{-1} \gamma(g h ; a)
\end{aligned}
$$

for all $a, b \in L, g, h \in G$.

In [Mas1, Section 1], Mastnak introduced $\mathrm{H}_{c}^{2}(F, K)$, the subgroup of $\mathrm{H}^{2}(F, K)$, generated by the cocycles with trivial multiplication part. That is,

$$
\mathrm{H}_{c}^{2}(F, K)=\mathrm{Z}_{c}^{2}(F, K) / \mathrm{B}_{c}^{2}(F, K)
$$

where

$$
\begin{aligned}
\mathrm{Z}_{c}^{2}(F, K) & =\left\{\tau \mid(\operatorname{triv}, \tau) \in \mathrm{Z}^{2}(F, K)\right\} \\
\mathrm{B}_{c}^{2}(F, K) & =\mathrm{B}^{2}(F, K) \cap \mathrm{Z}_{c}^{2}(F, K)
\end{aligned}
$$

Two sufficient conditions for $\mathrm{H}_{c}^{2}\left(k L, k^{G}\right)=\mathrm{H}^{2}\left(k L, k^{G}\right)$ were given in [Mas1, Theorem 4.4]. In particular, when $k$ is algebraically closed and $L$ is cyclic, $\mathrm{H}_{c}^{2}\left(k L, k^{G}\right)=$ $\mathrm{H}^{2}\left(k L, k^{G}\right)$.

In order to distinguish between two non-isomorphic Hopf algebras we will use their invariants, in particular Frobenius-Schur indicators and Grothendieck rings.

In [LM], Frobenius-Schur indicators were extended from groups to Hopf algebras as follows:

Definition $2.3([\mathrm{LM}])$. Let $\chi$ be a character of $H$. Define the $m$-th Frobenius-Schur indicator of $\chi$ via

$$
\nu_{m}(\chi):=\chi\left(\Lambda^{[m]}\right)=\sum \chi\left(\Lambda_{1} \cdots \Lambda_{m}\right)
$$

where $\Lambda$ is a (unique) integral in $H$ such that $\varepsilon(\Lambda)=1$ (such a $\Lambda$ exists by Maschke's theorem). 
Definition 2.4 ([Se] $)$. Let $K_{0}(H)^{+}$denote the abelian semigroup of all equivalence classes of representations of $H$ with the addition given by a direct sum. Then its enveloping group $K_{0}(H)$ has the structure of an ordered ring with involution * and is called the Grothendieck ring.

The structure of $K_{0}(H)$ was described in $\mathrm{NR}$. The multiplication in this ring is defined as follows: Let $\left[\pi_{1}\right]$ and $\left[\pi_{2}\right]$ denote the classes of representations equivalent to $\pi_{1}$ and $\pi_{2}$, then $\left[\pi_{1}\right] \bullet\left[\pi_{2}\right]$ is the class of the representation $\left(\pi_{1} \otimes \pi_{2}\right) \circ \Delta$; the unit of this ring is the class $[\varepsilon]$ and $[\pi]^{*}$ is the equivalence class of the dual representation ${ }^{t}(\pi \circ S)$ defined by $\left\langle{ }^{t}(\pi \circ S(h))(f), v\right\rangle=\langle f,(\pi \circ S(h))(v)\rangle$. The equivalence classes of irreducible representations of $H$ form a basis of $K_{0}(H)$. Moreover, $\left[\pi_{1}\right]^{*}=\left[\pi_{2}\right]$ if and only if $[\varepsilon]$ is a summand in the basis decomposition of $\left[\pi_{1}\right] \bullet\left[\pi_{2}\right]$ (necessarily of multiplicity 1). For simplicity of notation we will write $\pi$ instead of $[\pi]$ for elements of $K_{0}(H)$. In this paper, all irreducible representations of Hopf algebras under consideration will be 1- or 2-dimensional. We will not completely describe the structure of Grothendieck rings; it would be enough for our purposes to compute $\pi^{2}$ for each 2-dimensional irreducible representation $\pi$ of $H$.

\section{Hopf Algebras of Dimension $2^{m}$ With COMmutative Hopf subalgebra OF DIMENSION $2^{m-1}$.}

Let $H$ be a semisimple Hopf algebra of dimension $2^{m}$ over an algebraically closed field $k$ of characteristic 0 and assume that it has a commutative Hopf subalgebra $K$ of dimension $2^{m-1}$. Then, by the works of Masuoka (see [Ma1] and [Ma4]) or by [K2, Proposition 2.4], $H$ is equivalent to the bicrossed product $K \#_{\sigma}^{\tau} F$ of $K=k^{G}=(k G)^{*}$ where $G$ is a group of order $2^{m-1}$ and $F=k L=k\langle t\rangle \cong k \mathbb{Z}_{2}$ with an action $\rightarrow: F \otimes K \rightarrow K$, trivial coaction, a cocycle $\sigma: F \otimes F \rightarrow K$ and a dual cocycle $\tau: F \rightarrow K \otimes K$ (that is, $\left.(\sigma, \tau) \in \mathrm{Z}^{2}\left(k L, k^{G}\right)\right)$, and the action by $t$ being a Hopf algebra automorphism of $K$.

Since $\sigma$ and $\tau$ are normalized they are completely determined by $\sigma(t, t)$ and $\tau(t)$. We can write $\sigma(t, t)$ and $\tau(t)$ in terms of the basis for $(k G)^{*}$ :

$$
\begin{aligned}
\sigma(t, t) & =\sum_{g \in G} \sigma_{t}(g) p_{g} \\
\tau(t) & =\sum_{g, h \in G} \tau_{t}(g, h) p_{g} \otimes p_{h}
\end{aligned}
$$

where $\sigma_{t}(g)=\sigma(g ; t, t), \tau_{t}(g, h)=\tau(g, h ; t) \in k$. Since $(\sigma, \tau) \in \mathrm{Z}^{2}\left(k L, k^{G}\right), \sigma_{t}$ and $\tau_{t}$ satisfy the following properties for any $g, h \in G$ :

$$
\begin{aligned}
\sigma_{t}(1) & =1 \\
\tau_{t}(1, h) & =\tau_{t}(g, 1)=1 \\
\sigma_{t}(t \rightarrow g) & =\sigma_{t}(g)
\end{aligned}
$$




$$
\begin{aligned}
\tau_{t}(g, h) \tau_{t}(g h, k) & =\tau_{t}(g, h k) \tau_{t}(h, k) \\
\sigma_{t}(g h) & =\tau_{t}(g, h) \tau_{t}(t \rightarrow g, t \rightarrow h) \sigma_{t}(g) \sigma_{t}(h)
\end{aligned}
$$

By the definition of $\mathrm{B}^{2}\left(k L, k^{G}\right)$, we get the following lemma (note that $\gamma$ from the lemma corresponds to $\gamma\left({ }_{-}, t\right)$ from the definition of $\mathrm{B}^{2}\left(k L, k^{G}\right)$ in Section 2):

Lemma 3.1. Let $\gamma \in\left(k^{G}\right)^{\times}$and $\gamma(1)=1$. Then the extension $H=(k G)^{*} \#_{\sigma}^{\tau} k L$ is equivalent to the extension $H_{\gamma}=(k G)^{*} \#_{\gamma_{\sigma}}^{\gamma^{-1}} k L$, where

$$
\begin{array}{r}
\left({ }^{\gamma} \sigma\right)_{t}(g)=\gamma(g) \gamma(t \rightarrow g) \sigma_{t}(g) \\
\left(\tau^{\gamma^{-1}}\right)_{t}(g, h)=\gamma(g h)(\gamma(g) \gamma(h))^{-1} \tau_{t}(g, h)
\end{array}
$$

Remark 3.2. Since $k$ is algebraically closed and $L$ is cyclic, by Mac, Chapter IV, Theorem 7.1], $\mathrm{H}^{2}\left(L,\left(k^{G}\right)^{\times}\right)=0$ and therefore $\sigma \in \mathrm{B}^{2}\left(L,\left(k^{G}\right)^{\times}\right)$. Then $(\sigma)_{t}(g)=$ $\gamma(g) \gamma(t \rightarrow g)$ for some $\gamma \in\left(k^{G}\right)^{\times}$with $\gamma(1)=1$. Thus $(k G)^{*} \#_{\sigma}^{\tau} k L$ is equivalent to $(k G)^{*} \#^{\tau^{\gamma}} k L$. Therefore in this case Lemma3.1] implies the result of [Mas1, Theorem $4.4(1)]$ that $\mathrm{H}^{2}\left(k L, k^{G}\right)=\mathrm{H}_{c}^{2}\left(k L, k^{G}\right)$.

Definition 3.3. We will say that two actions of $L$ on $G, \rightarrow_{1}$ and $\rightarrow_{2}$, are similar if there is a group automorphism $f: G \longrightarrow G$ such that $f\left(t \rightarrow_{1} g\right)=t \rightarrow_{2} f(g)$ for all $g \in G$.

Remark 3.4. Since $|L|=2$, the number of non-similar non-trivial actions of $L$ on $G$ equals the number of distinct conjugacy classes of elements of order 2 in $\operatorname{Aut}(\mathbf{G})$.

The following result is contained implicitly in the works by Masuoka (see, for example Ma1] or [Ma3]). We give the proof for completeness.

Lemma 3.5. Let $F=k\langle t\rangle \cong k \mathbb{Z}_{2}$ and $K=k^{G}$ for a finite group $G$. Consider nontrivial Hopf algebras $H_{1}=K \#_{\sigma_{1}}^{\tau_{1}} F$ and $H_{2}=K \#_{\sigma_{2}}^{\tau_{2}} F$. Assume that the coactions are trivial and the corresponding actions, $\rightarrow_{1}$ and $\rightarrow_{2}$, by $t$ are group automorphisms of $G$. Then

(1) If $\rightarrow_{1}$ and $\rightarrow_{2}$ are similar with $f\left(t \rightarrow_{1} g\right)=t \rightarrow_{2} f(g),\left(\sigma_{1}\right)_{t}(g)=\left(\sigma_{2}\right)_{t}(f(g))$, and $\left(\tau_{1}\right)_{t}(g, h)=\left(\tau_{2}\right)_{t}(f(g), f(h))$ for all $g, h \in G$ then $H_{1}$ and $H_{2}$ are isomorphic.

(2) If $G$ is abelian and $H_{1}$ and $H_{2}$ are isomorphic then $\rightarrow_{1}$ and $\rightarrow_{2}$ are similar.

Proof. (1) Define

$$
\begin{aligned}
\mathcal{F}: k^{G} \#_{\sigma_{1}}^{\tau_{1}} k L & \longrightarrow k^{G} \#_{\sigma_{2}}^{\tau_{2}} k L \quad \text { by } \\
\mathcal{F}\left(p_{g} \# t\right) & =p_{f(g)} \# t
\end{aligned}
$$

It is straightforward to check that $\mathcal{F}$ is a Hopf algebra isomorphism. 
(2) Since $G$ is abelian, $K=k^{G}=k \hat{G}$ and therefore $\hat{G} \subseteq \mathbf{G}\left(H_{1}\right)=\mathbf{G}\left(H_{2}\right)$. Since $\left[H_{1}: K\right]=\left[H_{2}: K\right]=\operatorname{dim} F=2$ and $H_{1}$ and $H_{2}$ are nontrivial, $\hat{G}=\mathbf{G}\left(H_{1}\right)=\mathbf{G}\left(H_{2}\right)$ and $K=k \widehat{\mathbf{G}\left(H_{1}\right)}=k \widehat{\mathbf{G}\left(H_{2}\right)}$. Group-like elements are sent to group-like elements by Hopf algebra maps and therefore $K$ is preserved under any Hopf isomorphism $\mathcal{F}: H \longrightarrow H$. Then $\mathcal{F}$ restricts to an automorphism $\mathcal{F}_{k^{G}}: k^{G} \longrightarrow k^{G}$ and induces a group automorphism $f: G \longrightarrow G$ via $\mathcal{F}\left(p_{g}\right)=p_{f^{-1}(g)}$. Denote $1 \# t=\bar{t}$ and $p_{g} \# t=p_{g} \bar{t}$. Then

$$
\begin{aligned}
\mathcal{F}\left(\bar{t}_{g}\right) & =\mathcal{F}\left(p_{t \rightarrow_{1} g} \bar{t}\right)=\mathcal{F}\left(p_{t \rightarrow_{1} g}\right) \mathcal{F}(\bar{t})=p_{f^{-1}\left(t \rightarrow_{1} g\right)} \mathcal{F}(\bar{t}) \\
\mathcal{F}(\bar{t}) \mathcal{F}\left(p_{g}\right) & =\mathcal{F}(\bar{t}) p_{f^{-1}(g)}=p_{t-_{2} f^{-1}(g)} \mathcal{F}(\bar{t}) \\
\text { Thus } p_{f^{-1}\left(t \rightarrow_{1} g\right)} & =p_{t \rightarrow_{2} f^{-1}(g)} \text { and therefore } f^{-1}\left(t \rightarrow_{1} g\right)=t \rightarrow_{2} f^{-1}(g) .
\end{aligned}
$$

Let us now fix an action of $L$ on $G$. By [Mas1, Theorem 4.4 (1)] (or Remark 3.2), every extension is equivalent to an extension with a trivial cocycle. We will identify the equivalence class of $(k G)^{*} \#^{\tau} k L$ with $[(\operatorname{triv}, \tau)]=[\tau] \in \mathrm{H}_{c}^{2}\left(k L, k^{G}\right)$, where $\tau \in \mathrm{Z}_{c}^{2}\left(k L, k^{G}\right)$ satisfies

$$
\begin{aligned}
\tau_{t}(1, h) & =\tau_{t}(g, 1)=1 \\
\tau_{t}(g, h) \tau_{t}(g h, k) & =\tau_{t}(g, h k) \tau_{t}(h, k) \\
1 & =\tau_{t}(g, h) \tau_{t}(t \rightarrow g, t \rightarrow h)
\end{aligned}
$$

Thus our extension is completely determined by the cocycle $\tau_{t} \in \mathrm{Z}^{2}\left(G, k^{\times}\right)$which satisfies property (3.9) .

$L$ acts on $\mathrm{Z}^{2}\left(G, k^{\times}\right)$via

$$
(t \rightarrow \alpha)(g, h)=\alpha(t \rightarrow g, t \rightarrow h)
$$

This action induces an action of $L$ on $\mathrm{H}_{c}^{2}\left(k L, k^{G}\right)$.

Consider the Schur multiplier

$$
M(G)=\mathrm{H}^{2}\left(G, k^{\times}\right) .
$$

Then $L$ acts on $M(G)$ via

$$
t \rightarrow[\alpha]=[t \rightarrow \alpha]
$$

Consider the subgroup $M(G)^{-}$of $M(G)$ defined by

$$
M(G)^{-}=\left\{[\alpha] \in M(G) \mid t \rightarrow[\alpha]=[\alpha]^{-1}\right\}=\{[\alpha] \in M(G) \mid[\alpha](t \rightarrow[\alpha])=1\} .
$$

Theorem 3.6. Let $G$ be a group of order $2^{m-1}$ and $L=\langle t\rangle \cong \mathbb{Z}_{2}$ as in the beginning of this section. Consider the map

$$
\begin{aligned}
\Psi: \mathrm{H}^{2}\left(k L, k^{G}\right)=\mathrm{H}_{c}^{2}\left(k L, k^{G}\right) & \longrightarrow M(G) \\
\Psi([(\operatorname{triv}, \tau)])=\Psi([\tau]) & =\left[\tau_{t}\right]
\end{aligned}
$$

Then

(1) $\Psi$ is a well-defined group homomorphism. 
(2) $\operatorname{Im} \Psi \subseteq M(G)^{-}$.

(3) $\operatorname{Ker} \Psi \cong \hat{G}^{L} / N_{t}(\hat{G}) \cong \mathrm{H}^{2}(L, \hat{G})$.

Proof. Consider $\tau \in \mathrm{Z}_{c}^{2}\left(k L, k^{G}\right)$. Then, by (3.7) and (3.8), $\tau_{t} \in \mathrm{Z}^{2}\left(G, k^{\times}\right)$. If $\tau \in \mathrm{B}_{c}^{2}\left(k L, k^{G}\right)$ then $\tau_{t}(g, h)=\gamma_{t}(g)^{-1} \gamma_{t}(h)^{-1} \gamma_{t}(g h)$ and therefore $\tau_{t} \in \mathrm{B}^{2}\left(G, k^{\times}\right)$. Thus $\Psi$ is well-defined.

$\Psi$ is a homomorphism since $\left(\tau \tau^{\prime}\right)_{t}=\tau_{t} \tau_{t}^{\prime}$.

If $[\tau] \in \mathrm{H}_{c}^{2}\left(k L, k^{G}\right)$ then $\tau_{t}(g, h) \tau_{t}(t \rightarrow g, t \rightarrow h)=1$ by (3.9) and therefore

$$
\left[\tau_{t}\right]\left(t \rightarrow\left[\tau_{t}\right]\right)=\left[t \rightarrow \tau_{t}\right]=1
$$

Assume now that $[(\operatorname{triv}, \tau)]=[\tau] \in \operatorname{Ker} \Psi$. Then $\tau_{t} \in \mathrm{B}^{2}\left(G, k^{\times}\right)$and therefore $\tau_{t}(g, h)=\gamma(g) \gamma(h) \gamma(g h)^{-1}$ for some $\gamma \in\left(k^{G}\right)^{\times}$with $\gamma(1)=1$. Then $[(\operatorname{triv}, \tau)]=$ $[(\sigma$, triv $)] \in \mathrm{H}^{2}\left(k L, k^{G}\right)$ where $\sigma={ }^{\gamma}$ triv, that is,

$$
\sigma_{t}(g)=\gamma(g) \gamma(t \rightarrow g) .
$$

Formulas (3.2), (3.4), and (3.6) imply that $\sigma$ satisfies the following properties

$$
\begin{aligned}
\sigma_{t}(1) & =1 \\
\sigma_{t}(t \rightarrow g) & =\sigma_{t}(g) \\
\sigma_{t}(g h) & =\sigma_{t}(g) \sigma_{t}(h)
\end{aligned}
$$

Therefore $\sigma_{t} \in \hat{G}^{L}$ the subgroup of $\hat{G}$, fixed by $L$. Then $\sigma=\sigma^{\beta}$ for some $\beta \in \hat{G}^{L}$, where $\left(\sigma^{\beta}\right)_{t}=\beta$. Define a map

$$
\phi: \hat{G}^{L} \longrightarrow \operatorname{Ker} \Psi \quad \text { via } \phi(\beta)=\left[\left(\sigma^{\beta}, \text { triv }\right)\right]
$$

Since $\beta \in \hat{G}^{L},\left(\sigma^{\beta}\right.$, triv) satisfies properties (3.2)-(3.6) and therefore $\left(\sigma^{\beta}\right.$, triv) $\in$ $Z^{2}\left(k L, k^{G}\right)$. Since $\mathrm{H}^{2}\left(L,\left(k^{G}\right)^{\times}\right)=0,\left(\sigma^{\beta}\right)_{t}(g)=\gamma(g) \gamma(t \rightarrow g)$ for some $\gamma \in$ $\left(k^{G}\right)^{\times}$with $\gamma(1)=1$. Therefore $\left[\left(\sigma^{\beta}\right.\right.$, triv $\left.)\right]=\left[\left(\operatorname{triv}, \operatorname{triv}^{\gamma}\right)\right]$ and

$$
\Psi\left(\left[\left(\sigma^{\beta}, \text { triv }\right)\right]\right)=\Psi\left(\left[\left(\operatorname{triv}, \operatorname{triv}^{\gamma}\right)\right]\right)=\left[\operatorname{triv}^{\gamma}\right]=1
$$

Thus $\phi$ is well-defined. Since $\sigma^{\beta_{1} \beta_{2}}=\sigma^{\beta_{1}} \sigma^{\beta_{2}}, \phi$ is a homomorphism.

Since we have already shown that for every $[(\operatorname{triv}, \tau)] \in \operatorname{Ker} \Psi$ there exists $\beta \in \hat{G}^{L}$ such that $[(\operatorname{triv}, \tau)]=\left[\left(\sigma^{\beta}\right.\right.$, triv $\left.)\right], \phi$ is surjective. Moreover

$$
\begin{aligned}
\operatorname{Ker} \phi & =\left\{\beta \in \hat{G}^{L} \mid \beta=\gamma(t \rightarrow \gamma) \text { and } \gamma(g) \gamma(h) \gamma(g h)^{-1}=1\right\} \\
& =\{\gamma(t \rightarrow \gamma) \mid \gamma \in \hat{G}\}=N_{t}(\hat{G})
\end{aligned}
$$

Thus $\operatorname{Ker} \Psi \cong \hat{G}^{L} / N_{t}(\hat{G}) \cong \mathrm{H}^{2}(L, \hat{G})$.

Remark 3.7. Since $t^{2}=1$, the map $\Psi$ is the coconnecting homomorphism defined in [Mas1, Section 6]. 
Corollary 3.8. Let $G$ be a group of order $2^{m-1}$ and $L$ be a group of order 2 . For any given action $\rightarrow$ of $L$ on $G,\left|\mathrm{H}^{2}\left(k L, k^{G}\right)\right| \leq\left|\mathrm{H}^{2}(L, \hat{G})\right| \cdot\left|M(G)^{-}\right|$.

Corollary 3.9. Let $\mathbf{G}$ be an abelian group of order $2^{m-1}$ and let $N$ be the number of distinct conjugacy classes of elements of order 2 in $\operatorname{Aut}(\mathbf{G})$. Then there are at most $N(|\mathbf{G}| \cdot|M(\mathbf{G})| / 2-1)$ nonisomorphic Hopf algebras of dimension $2^{m}$ with group of group-like elements isomorphic to $\mathbf{G}$.

Proof. Every non-trivial Hopf algebra under consideration is equivalent to $H=$ $k^{G} \#^{\tau} k L$ where $G=\widehat{\mathbf{G}}$ and $|L|=2$. Therefore the action of $L$ on $G$ is nontrivial and corresponds to an automorphism of $G$ of order 2. Moreover, $\hat{G}^{L}$ is a proper subgroup of $\hat{G}$ and therefore

$$
\left|\mathrm{H}^{2}(L, \hat{G})\right| \leq\left|\hat{G}^{L}\right| \leq|\hat{G}| / 2=|\mathbf{G}| / 2
$$

\section{Hopf algebras of Dimension $2^{2 n+1}$ With $\mathbf{G}(H)=\mathbb{Z}_{2^{n}} \times \mathbb{Z}_{2^{n}}$}

In this section we describe, up to equivalence, all possible non-commutative semisimple Hopf algebras of dimension $2^{2 n+1}$ with group of group-like elements isomorphic to $\mathbb{Z}_{2^{n}} \times \mathbb{Z}_{2^{n}}$ for $n \geq 2$.

Let $H$ be a nontrivial semisimple Hopf algebra of dimension $2^{2 n+1}$ with $\mathbf{G}(H) \cong$ $\mathbb{Z}_{2^{n}} \times \mathbb{Z}_{2^{n}}$. Then, as in Section [3, $H$ is equivalent to the bicrossed product $K \#_{\sigma}^{\tau} F$ of $K=k \mathbf{G}(H)=(k G)^{*}$ where $G=\widehat{\mathbf{G}(H)} \cong \mathbb{Z}_{2^{2 n}} \times \mathbb{Z}_{2^{2 n}}$ and $F=k L=k\langle t\rangle \cong k \mathbb{Z}_{2}$ with an action $\rightarrow: F \otimes K \rightarrow K$ by Hopf algebra automorphisms, trivial coaction, a cocycle $\sigma: F \otimes F \rightarrow K$, and a dual cocycle $\tau: F \rightarrow K \otimes K$.

Let $G=\langle x\rangle \times\langle y\rangle \cong \mathbb{Z}_{2^{n}} \times \mathbb{Z}_{2^{n}}$. Then $\hat{G}=\mathbf{G}(H)=\left\langle\beta_{1}\right\rangle \times\left\langle\beta_{2}\right\rangle \cong \mathbb{Z}_{2^{n}} \times \mathbb{Z}_{2^{n}}$ where

$$
\beta_{1}\left(x^{a} y^{b}\right)=\xi^{a} \quad \beta_{2}\left(x^{a} y^{b}\right)=\xi^{b}
$$

and $\xi$ is a primitive $2^{n}$-th root of unity.

Define

$$
\alpha_{p, q}\left(x^{a_{1}} y^{a_{2}}, x^{b_{1}} y^{b_{2}}\right)=\xi^{a_{p} b_{q}}
$$

Then $M(G)=\left\{\left[\alpha_{1,2}^{k}\right] \mid k \in \mathbb{Z}_{2^{n}}\right\}=\left\langle\left[\alpha_{1,2}\right]\right\rangle \cong \mathbb{Z}_{2^{n}}$ (see [Ka, Corollary 2.2.12]).

Define $\tau_{p, q}^{k}$ via

$$
\left(\tau_{p, q}^{k}\right)_{t}=\alpha_{p, q}^{k}
$$

Then $\tau_{p, q}^{k}$ is a dual cocycle.

Proposition 4.1. (1) In $M(G)$

$$
\begin{aligned}
& {\left[\alpha_{2,1}\right]=\left[\alpha_{1,2}^{-1}\right]} \\
& {\left[\alpha_{j, j}\right]=1}
\end{aligned}
$$

where $j=1,2$. 
(2) If $t \rightarrow x=x y^{r}$ and $t \rightarrow y=y^{s}$ then in $\mathrm{H}^{2}\left(k L, k^{G}\right),\left[\left(\sigma^{\beta_{1}}\right.\right.$, triv $\left.)\right]=\left[\left(\operatorname{triv}, \tau^{\prime}\right)\right]$ where $\tau_{t}^{\prime}=\alpha^{\prime}$ for $\alpha^{\prime}$ defined via

$$
\alpha^{\prime}\left(x^{a_{1}} y^{a_{2}}, x^{b_{1}} y^{b_{2}}\right)=(-1)^{k}
$$

with $k=0$ if $0 \leq a_{1}+b_{1}<2^{n}$ and $k=1$ if $2^{n} \leq a_{1}+b_{1}<2^{n+1}$.

(3) If $t \rightarrow x=x^{q} y^{2^{n-1} r}$ and $t \rightarrow y=y^{-1}$ then in $\mathrm{H}^{2}\left(k L, k^{G}\right)$,

$$
\left[\left(\sigma^{\beta_{2}^{2^{n-1}}}, \text { triv }\right)\right]=\left[\left(\operatorname{triv}, \tau_{2,2}^{2^{n-1}}\right)\right] .
$$

(4) If $t \rightarrow x=x^{-1}$ and $t \rightarrow y=y^{s}$ then in $\mathrm{H}^{2}\left(k L, k^{G}\right)$,

$$
\left[\left(\sigma^{\beta_{1}^{2^{n-1}}}, \operatorname{triv}\right)\right]=\left[\left(\operatorname{triv}, \tau_{1,1}^{2^{n-1}}\right)\right] .
$$

Proof. Let $j \in\{1,2\}$ and let $w$ be a primitive $2^{n+1}$-th root of 1 , such that $\omega^{2}=-\xi$. Define $\mu, \gamma_{j}, \eta \in\left(k^{G}\right)^{\times}$via

$$
\begin{aligned}
\mu\left(x^{a_{1}} y^{a_{2}}\right) & =\xi^{a_{1} a_{2}} \\
\gamma_{j}\left(x^{a_{1}} y^{a_{2}}\right) & =\omega^{a_{j}^{2}} \\
\eta\left(x^{a_{1}} y^{a_{2}}\right) & =\omega^{a_{1}}
\end{aligned}
$$

Then

$$
\begin{aligned}
\alpha_{2,1}\left(x^{a_{1}} y^{a_{2}}, x^{b_{1}} y^{b_{2}}\right) \mu\left(x^{a_{1}} y^{a_{2}}\right) \mu\left(x^{b_{1}} y^{b_{2}}\right) \mu\left(x^{a_{1}} y^{a_{2}} x^{b_{1}} y^{b_{2}}\right)^{-1} & =\xi^{a_{2} b_{1}} \xi^{a_{1} a_{2}} \xi^{b_{1} b_{2}} \xi^{-\left(a_{1}+b_{1}\right)\left(a_{2}+b_{2}\right)} \\
& =\xi^{-a_{1} b_{2}}=\alpha_{1,2}^{-1}\left(x^{a_{1}} y^{a_{2}}, x^{b_{1}} y^{b_{2}}\right) \\
\gamma_{j}\left(x^{a_{1}} y^{a_{2}}\right) \gamma_{j}\left(x^{b_{1}} y^{b_{2}}\right) \gamma_{j}^{-1}\left(x^{a_{1}} y^{a_{2}} x^{b_{1}} y^{b_{2}}\right) & =\omega^{a_{j}^{2}} \omega^{b_{j}^{2}} \omega^{-\left(a_{j}+b_{j}-2^{n} k\right)^{2}} \\
& =\xi^{a_{j} b_{j}}=\alpha_{j, j}\left(x^{a_{1}} y^{a_{2}}, x^{b_{1}} y^{b_{2}}\right)
\end{aligned}
$$

where $k=0$ if $0 \leq a_{j}+b_{j}<2^{n}$ and $k=1$ if $2^{n} \leq a_{j}+b_{j}<2^{n+1}$.

(2) Assume now that $t \rightarrow x=x y^{r}$ and $t \rightarrow y=y^{s}$. Then $\beta_{1} \in \hat{G}^{L}$ and $\left[\left(\sigma^{\beta_{1}}\right.\right.$, triv $\left.)\right] \in \mathrm{H}^{2}\left(k L, k^{G}\right)$. We will show that $\left[\left(\sigma^{\beta_{1}}, \operatorname{triv}\right)\right]=\left[\left(\operatorname{triv}, \tau^{\prime}\right)\right]$ where $\tau_{t}^{\prime}=\alpha^{\prime}$ with the notation $\sigma=\sigma^{\beta_{1}}$ and $\tau=$ triv:

$$
\begin{aligned}
\left({ }^{\eta} \sigma\right)_{t}\left(x^{a_{1}} y^{a_{2}}\right) & =\eta\left(x^{a_{1}} y^{a_{2}}\right) \eta\left(t \rightarrow x^{a_{1}} y^{a_{2}}\right) \sigma_{t}\left(x^{a_{1}} y^{a_{2}}\right) \\
& =\eta\left(x^{a_{1}} y^{a_{2}}\right) \eta\left(x^{a_{1}} y^{r a_{1}+s a_{2}}\right) \beta_{1}\left(x^{a_{1}} y^{a_{2}}\right) \\
& =\omega^{a_{1}} \omega^{a_{1}} \xi^{a_{1}}=1 \\
\left(\tau^{\eta^{-1}}\right)_{t}\left(x^{a_{1}} y^{a_{2}}, x^{b_{1}} y^{b_{2}}\right) & =\eta\left(x^{a_{1}} y^{a_{2}} x^{b_{1}} y^{b_{2}}\right) \eta^{-1}\left(x^{a_{1}} y^{a_{2}}\right) \eta^{-1}\left(x^{b_{1}} y^{b_{2}}\right) \\
& =\omega^{a_{1}+b_{1}-2^{n} k} \omega^{-a_{1}} \omega^{-b_{1}}=(-1)^{k} \\
& =\alpha^{\prime}\left(x^{a_{1}} y^{a_{2}}, x^{b_{1}} y^{b_{2}}\right)
\end{aligned}
$$

where $k=0$ if $0 \leq a_{1}+b_{1}<2^{n}$ and $k=1$ if $2^{n} \leq a_{1}+b_{1}<2^{n+1}$. 
(3) Assume now that $t \rightarrow x=x^{q} y^{2^{n-1} r}$ and $t \rightarrow y=y^{-1}$. Then $\beta_{2}^{2^{n-1}} \in$ $\hat{G}^{L}$ and $\left[\left(\sigma^{\beta_{2}^{2 n-1}}, \operatorname{triv}\right)\right] \in \mathrm{H}^{2}\left(k L, k^{G}\right)$. We will show that $\left[\left(\sigma^{\beta_{2}^{2 n-1}}, \operatorname{triv}\right)\right]=$ $\left[\left(\operatorname{triv}, \tau_{2,2}^{2^{n-1}}\right)\right]$. Denote $\sigma=\sigma_{2}^{\beta_{2}^{n-1}}, \tau=$ triv and $\gamma=\gamma_{2}^{2^{n-1}}$. Then

$$
\begin{aligned}
\left({ }^{\gamma} \sigma\right)_{t}\left(x^{a_{1}} y^{a_{2}}\right) & =\gamma\left(x^{a_{1}} y^{a_{2}}\right) \gamma\left(t \rightarrow x^{a_{1}} y^{a_{2}}\right) \sigma_{t}\left(x^{a_{1}} y^{a_{2}}\right) \\
& =\gamma\left(x^{a_{1}} y^{a_{2}}\right) \gamma\left(x^{q a_{1}} y^{2^{n-1}} r a_{1}-a_{2}\right) \beta_{2}^{2^{n-1}}\left(x^{a_{1}} y^{a_{2}}\right) \\
& =i^{a_{2}^{2}} i^{\left(2^{n-1} r a_{1}-a_{2}\right)^{2}}(-1)^{a_{2}}=1 \\
\left(\tau^{\gamma^{-1}}\right)_{t}\left(x^{a_{1}} y^{a_{2}}, x^{b_{1}} y^{b_{2}}\right) & =\gamma\left(x^{a_{1}} y^{a_{2}} x^{b_{1}} y^{b_{2}}\right) \gamma^{-1}\left(x^{a_{1}} y^{a_{2}}\right) \gamma^{-1}\left(x^{b_{1}} y^{b_{2}}\right) \\
& =i^{\left(a_{2}+b_{2}\right)^{2}-a_{2}^{2}-b_{2}^{2}}=(-1)^{a_{2} b_{2}}
\end{aligned}
$$

(4) Part 4 is proved similarly to part 3, using $\gamma=\gamma_{1}^{2^{n-1}}$.

Since $H$ is noncommutative, the action $\rightarrow$ is not trivial, and $t$ acts on $G$ as a group automorphism of order 2 . Let

$$
\begin{aligned}
& t \rightarrow x=x^{a} y^{b} \\
& t \rightarrow y=x^{c} y^{d}
\end{aligned}
$$

This action corresponds to $A=\left[\begin{array}{ll}a & c \\ b & d\end{array}\right] \in G L_{2}\left(\mathbb{Z}_{2^{n}}\right)$ such that $A \neq I$ and $A^{2}=I$. Two actions are similar if and only if the corresponding matrices are similar.

Theorem 4.2. For $n \geq 3$, there are 16 non-similar actions of $t$ on $G$, corresponding to the matrices in (4.1)- 4.4). Only 6 of these actions are non-similar when $n=2$.

Proof. Consider an action which corresponds to $A=\left[\begin{array}{ll}a & c \\ b & d\end{array}\right] \in G L_{2}\left(\mathbb{Z}_{2^{n}}\right)$. Since $A^{2}=I$

$$
\begin{array}{ll}
a^{2}+b c=1 & (a+d) c=0 \\
d^{2}+b c=1 & (a+d) b=0
\end{array}
$$

Therefore $a$ and $d$ have the same parity and $(a+d)(a-d)=0$.

(1) Assume that $a$ and $d$ are both even. Then $b$ and $c$ are both odd and $d=-a$. The matrix $\left[\begin{array}{ll}1 & 0 \\ a & c\end{array}\right]$ is invertible and

$$
\left[\begin{array}{ll}
1 & 0 \\
a & c
\end{array}\right]\left[\begin{array}{ll}
a & c \\
b & -a
\end{array}\right]=\left[\begin{array}{ll}
0 & 1 \\
1 & 0
\end{array}\right]\left[\begin{array}{ll}
1 & 0 \\
a & c
\end{array}\right]
$$

Thus all matrices of this type lie in one conjugacy class with representative

$$
\left[\begin{array}{ll}
0 & 1 \\
1 & 0
\end{array}\right]
$$


(2) Assume that $a$ and $d$ are both odd and $a+d=2 q$ in $\mathbb{Z}_{2^{n}}$, where $q$ is odd.

Then $b, c \in\left\{0,2^{n-1}\right\}$ and $a^{2}=d^{2}=1$. Therefore $a, d \in\left\{ \pm 1, \pm 1+2^{n-1}\right\}$. Thus either $d=a$ or $d=a+2^{n-1}$ (the latter case is possible only for $n \geq 3$, since when $n=2, a+(a+2)=0)$. Since

$$
\begin{aligned}
{\left[\begin{array}{ll}
0 & 1 \\
1 & 0
\end{array}\right]\left[\begin{array}{ll}
a & c \\
b & d
\end{array}\right] } & =\left[\begin{array}{ll}
d & b \\
c & a
\end{array}\right]\left[\begin{array}{ll}
0 & 1 \\
1 & 0
\end{array}\right] \\
{\left[\begin{array}{ll}
1 & 1 \\
0 & 1
\end{array}\right]\left[\begin{array}{ll}
a & 2^{n-1} \\
2^{n-1} & a
\end{array}\right] } & =\left[\begin{array}{ll}
a+2^{n-1} & 0 \\
2^{n-1} & a+2^{n-1}
\end{array}\right]\left[\begin{array}{ll}
1 & 1 \\
0 & 1
\end{array}\right] \\
{\left[\begin{array}{ll}
1 & 1 \\
1 & 0
\end{array}\right]\left[\begin{array}{ll}
a & 0 \\
2^{n-1} & a+2^{n-1}
\end{array}\right] } & =\left[\begin{array}{ll}
a+2^{n-1} & 0 \\
0 & a
\end{array}\right]\left[\begin{array}{ll}
1 & 1 \\
1 & 0
\end{array}\right]
\end{aligned}
$$

every matrix of this type is similar to one of the following 11 matrices:

$$
\left[\begin{array}{ll}
-1 & 0 \\
0 & -1
\end{array}\right] ; \quad\left[\begin{array}{ll}
1+2^{n-1} & 0 \\
0 & 1+2^{n-1}
\end{array}\right] ; \quad\left[\begin{array}{cc}
-1+2^{n-1} & 0 \\
0 & -1+2^{n-1}
\end{array}\right]
$$

$\left[\begin{array}{cc}1 & 0 \\ 2^{n-1} & 1\end{array}\right] ; \quad\left[\begin{array}{cc}-1 & 0 \\ 2^{n-1} & -1\end{array}\right] ; \quad\left[\begin{array}{cc}1+2^{n-1} & 0 \\ 2^{n-1} & 1+2^{n-1}\end{array}\right] ; \quad\left[\begin{array}{cc}-1+2^{n-1} & 0 \\ 2^{n-1} & -1+2^{n-1}\end{array}\right]$;

$\left[\begin{array}{cc}1 & 0 \\ 0 & 1+2^{n-1}\end{array}\right] ; \quad\left[\begin{array}{cc}-1 & 0 \\ 0 & -1+2^{n-1}\end{array}\right] ; \quad\left[\begin{array}{cc}1 & 2^{n-1} \\ 2^{n-1} & 1+2^{n-1}\end{array}\right] ; \quad\left[\begin{array}{cc}-1 & 2^{n-1} \\ 2^{n-1} & -1+2^{n-1}\end{array}\right]$

(3) Assume that $a$ and $d$ are both odd and $a+d=4 q$ in $\mathbb{Z}_{2^{n}}$.

If $n=2$ the assumption means that $a=-d$.

If $n \geq 3, a-d=2(a-2 q)$ where $a-2 q$ is odd. Since $(a+d)(a-d)=0$ in $\mathbb{Z}_{2^{n}}, a+d \in\left\{0,2^{n-1}\right\}$. Thus either $d=-a$ or $d=-a+2^{n-1}$. Therefore $a, d \in\left\{ \pm 1, \pm 1+2^{n-1}\right\}$.

(a) Assume that $b$ or $c$ is odd. Then $d=-a$ (since $(a+d) b=(a+d) c=$ $0)$. Without loss of generality, $b$ is odd. Then the matrix $\left[\begin{array}{ll}0 & 1 \\ b & -a\end{array}\right]$ is invertible and

$$
\left[\begin{array}{ll}
0 & 1 \\
b & -a
\end{array}\right]\left[\begin{array}{ll}
a & c \\
b & -a
\end{array}\right]=\left[\begin{array}{ll}
0 & 1 \\
1 & 0
\end{array}\right]\left[\begin{array}{ll}
0 & 1 \\
b & -a
\end{array}\right]
$$

Thus every matrix of this type is similar to $\left[\begin{array}{ll}0 & 1 \\ 1 & 0\end{array}\right]$.

(b) Assume that $b$ and $c$ are even and $d=-a$.

If $n=2$, every matrix of this type is similar to $\left[\begin{array}{ll}1 & 0 \\ 0 & -1\end{array}\right]$ or $\left[\begin{array}{ll}1 & 2 \\ 2 & -1\end{array}\right]$. Assume now that $n \geq 3$. Since $\left[\begin{array}{ll}a & c \\ b & -a\end{array}\right]$ is similar to $\left[\begin{array}{ll}-a & b \\ c & a\end{array}\right]$ we 
may assume that $a \equiv 1(\bmod 4)$. Then the matrices

$$
\left[\begin{array}{ll}
\frac{a+1}{2} & \frac{c}{2} \\
-\frac{b}{2} & \frac{a+1}{2}
\end{array}\right] \text { and }\left[\begin{array}{ll}
\frac{a+1}{2}+2^{n-2} & \frac{c}{2} \\
-\frac{b}{2} & \frac{a+1}{2}+2^{n-2}
\end{array}\right]
$$

are invertible. Since $a^{2}+b c=1, \frac{a^{2}+b c-1}{2}=0$ or $2^{n-1}$.

When $\frac{a^{2}+b c-1}{2}=0$

$$
\left[\begin{array}{ll}
\frac{a+1}{2} & \frac{c}{2} \\
-\frac{b}{2} & \frac{a+1}{2}
\end{array}\right]\left[\begin{array}{ll}
a & c \\
b & -a
\end{array}\right]=\left[\begin{array}{ll}
1 & 0 \\
0 & -1
\end{array}\right]\left[\begin{array}{ll}
\frac{a+1}{2} & \frac{c}{2} \\
-\frac{b}{2} & \frac{a+1}{2}
\end{array}\right]
$$

When $\frac{a^{2}+b c-1}{2}=2^{n-1}$

$$
\left[\begin{array}{ll}
\frac{a+1+2^{n-1}}{2} & \frac{c}{2} \\
-\frac{b}{2} & \frac{a+1+2^{n-1}}{2}
\end{array}\right]\left[\begin{array}{ll}
a & c \\
b & -a
\end{array}\right]=\left[\begin{array}{ll}
1+2^{n-1} & 0 \\
0 & -1+2^{n-1}
\end{array}\right]\left[\begin{array}{ll}
\frac{a+1+2^{n-1}}{2} & \frac{c}{2} \\
-\frac{b}{2} & \frac{a+1+2^{n-1}}{2}
\end{array}\right]
$$

Therefore every matrix of this type is similar to

$$
\left[\begin{array}{ll}
1 & 0 \\
0 & -1
\end{array}\right] \text { or }\left[\begin{array}{ll}
1+2^{n-1} & 0 \\
0 & -1+2^{n-1}
\end{array}\right]
$$

(c) Assume that $b$ and $c$ are even and $d=-a+2^{n-1}$. Then $n \geq 3$ and as in the previous case we may assume that $a \equiv 1(\bmod 4)$. Then the matrices

$$
\left[\begin{array}{ll}
\frac{a+1}{2} & \frac{c}{2} \\
-\frac{b}{2} & \frac{a+1}{2}
\end{array}\right] \text { and }\left[\begin{array}{ll}
\frac{a+1+2^{n-1}}{2} & \frac{c}{2} \\
-\frac{b}{2} & \frac{a+1+2^{n-1}}{2}
\end{array}\right]
$$

are invertible. Since $a^{2}+b c=1, \frac{a^{2}+b c-1}{2}=0$ or $2^{n-1}$.

When $\frac{a^{2}+b c-1}{2}=0$

$$
\begin{gathered}
{\left[\begin{array}{ll}
\frac{a+1+2^{n-1}}{2} & \frac{c}{2} \\
-\frac{b}{2} & \frac{a+1+2^{n-1}}{2}
\end{array}\right]\left[\begin{array}{ll}
a & c \\
b & -a
\end{array}\right]=\left[\begin{array}{ll}
1 & 0 \\
0 & -1+2^{n-1}
\end{array}\right]\left[\begin{array}{ll}
\frac{a+1+2^{n-1}}{2} & \frac{c}{2} \\
-\frac{b}{2} & \frac{a+1+2^{n-1}}{2}
\end{array}\right]} \\
\text { When } \frac{a^{2}+b c-1}{2}=2^{n-1} \\
{\left[\begin{array}{ll}
\frac{a+1}{2} & \frac{c}{2} \\
-\frac{b}{2} & \frac{a+1}{2}
\end{array}\right]\left[\begin{array}{ll}
a & c \\
b & -a
\end{array}\right]=\left[\begin{array}{ll}
1+2^{n-1} & 0 \\
0 & -1
\end{array}\right]\left[\begin{array}{ll}
\frac{a+1}{2} & \frac{c}{2} \\
-\frac{b}{2} & \frac{a+1}{2}
\end{array}\right]}
\end{gathered}
$$

Therefore every matrix of this type is similar to

$$
\left[\begin{array}{ll}
1 & 0 \\
0 & -1+2^{n-1}
\end{array}\right] \quad \text { or } \quad\left[\begin{array}{ll}
1+2^{n-1} & 0 \\
0 & -1
\end{array}\right]
$$

Hopf algebras associated with two different actions are isomorphic only if the corresponding matrices are similar. Thus we will consider the following 16 cases, corresponding to the matrices in (4.1)-(4.4) (only 6 of these cases are possible when $n=2)$ : 
Theorem 4.3. Assume that $H$ is a semisimple Hopf algebra of dimension $2^{2 n+1}$ with group of group-like elements isomorphic to $\mathbb{Z}_{2^{n}} \times \mathbb{Z}_{2^{n}}$ for $n \geq 2$, which corresponds to the action associated with one of the matrices in (4.1)-(4.4). Then, for $\Psi$ defined in Theorem 3.6, we have

$$
\begin{aligned}
\mathrm{H}^{2}\left(k L, k^{G}\right) & \cong \mathrm{H}_{c}^{2}\left(k L, k^{G}\right) \cong \operatorname{Ker} \Psi \times \operatorname{Im} \Psi \\
& \cong \hat{G}^{L} / N_{t}(\hat{G}) \times M(G)^{-} \cong \mathrm{H}^{2}(L, \hat{G}) \times M(G)^{-}
\end{aligned}
$$

In order to prove this theorem we will consider the following sixteen cases corresponding to the matrices in (4.1)-(4.4) (only six of them are possible when $n=2$ ) and establish the results in each of the cases separately.

4.1. Case 1: $t \rightarrow x=y, \quad t \rightarrow y=x$.

Proposition 4.4. Let $\omega$ be a primitive $2^{n+1}$-th root of 1 such that $\omega^{2}=\xi$.

$$
\mathrm{H}^{2}\left(k L, k^{G}\right)=\left\langle\left[\left(\sigma^{\beta}, \tau_{1,2}^{j}\right)\right]\right\rangle \cong \operatorname{Im} \Psi \cong\left\langle\left[\alpha_{1,2}^{\prime}\right]\right\rangle \cong \mathbb{Z}_{2^{n}}
$$

where

$$
\alpha_{1,2}^{\prime}\left(x^{a_{1}} y^{a_{2}}, x^{b_{1}} y^{b_{2}}\right)=\omega^{a_{1} b_{2}-a_{2} b_{1}}(-1)^{\left(a_{1}+b_{1}\right) k_{2}+\left(a_{2}+b_{2}\right) k_{1}}
$$

with $k_{j}=0$ if $0 \leq a_{j}+b_{j}<2^{n}$ and $k_{j}=1$ if $2^{n} \leq a_{j}+b_{j}<2^{n+1}$ and

$$
\beta\left(x^{a_{1}} y^{a_{2}}\right)=\xi^{a_{1} a_{2}} .
$$

Proof. Since $t \rightarrow x=y$ and $t \rightarrow y=x$,

$$
\begin{aligned}
t \rightarrow \alpha_{1,2} & =\alpha_{2,1} \\
t \rightarrow \beta_{1} & =\beta_{2} \\
t \rightarrow \beta_{2} & =\beta_{1}
\end{aligned}
$$

By part 1 of Proposition 4.1, $\left[\alpha_{2,1}\right]=\left[\alpha_{1,2}^{-1}\right]$ in $M(G)$. Therefore $M(G)^{-}=M(G)$. We will now define $\alpha_{1,2}^{\prime} \in \mathrm{Z}^{2}\left(G, k^{\times}\right)$such that $\left[\alpha_{1,2}\right]=\left[\alpha_{1,2}^{\prime}\right]$ and $\alpha_{1,2}^{\prime}\left(t \rightarrow \alpha_{1,2}^{\prime}\right)=1$.

Let $\omega$ be a primitive $2^{n+1}$-th root of 1 such that $\omega^{2}=\xi$. Define $\gamma \in\left(k^{G}\right)^{\times}$and $\alpha_{1,2}^{\prime} \in \mathrm{Z}^{2}\left(G, k^{\times}\right)$via

$$
\begin{aligned}
\gamma\left(x^{a_{1}} y^{a_{2}}\right) & =\omega^{a_{1} a_{2}} \\
\alpha_{1,2}^{\prime}(g, h) & =\alpha_{1,2}(g, h) \gamma(g) \gamma(h) \gamma(g h)^{-1}
\end{aligned}
$$

Then $\left[\alpha_{1,2}^{\prime}\right]=\left[\alpha_{1,2}\right]$ and

$$
\begin{aligned}
\alpha_{1,2}^{\prime}\left(x^{a_{1}} y^{a_{2}}, x^{b_{1}} y^{b_{2}}\right) & =\xi^{a_{1} b_{2}} \omega^{a_{1} a_{2}} \omega^{b_{1} b_{2}} \omega^{-\left(a_{1}+b_{1}-2^{n} k_{1}\right)\left(a_{2}+b_{2}-2^{n} k_{2}\right)} \\
& =\omega^{a_{1} b_{2}-a_{2} b_{1}}(-1)^{\left(a_{1}+b_{1}\right) k_{2}+\left(a_{2}+b_{2}\right) k_{1}}
\end{aligned}
$$

where $k_{j}=0$ if $0 \leq a_{j}+b_{j}<2^{n}$ and $k_{j}=1$ if $2^{n} \leq a_{j}+b_{j}<2^{n+1}$.

$$
\alpha_{1,2}^{\prime}\left(x^{a_{1}} y^{a_{2}}, x^{b_{1}} y^{b_{2}}\right)\left(t \rightarrow \alpha_{1,2}^{\prime}\right)\left(x^{a_{1}} y^{a_{2}}, x^{b_{1}} y^{b_{2}}\right)
$$




$$
\begin{aligned}
& =\xi^{a_{1} b_{2}} \omega^{a_{1} a_{2}} \omega^{b_{1} b_{2}} \omega^{-\left(a_{1}+b_{1}-2^{n} k_{1}\right)\left(a_{2}+b_{2}-2^{n} k_{2}\right)} \xi^{a_{2} b_{1}} \omega^{a_{2} a_{1}} \omega^{b_{2} b_{1}} \omega^{-\left(a_{2}+b_{2}-2^{n} k_{2}\right)\left(a_{1}+b_{1}-2^{n} k_{1}\right)} \\
& =\xi^{a_{1} b_{2}+a_{2} b_{1}} \xi^{a_{1} a_{2}} \xi^{b_{1} b_{2}} \xi^{-\left(a_{1}+b_{1}-2^{n} k_{1}\right)\left(a_{2}+b_{2}-2^{n} k_{2}\right)}=\xi^{a_{1} b_{2}+a_{2} b_{1}} \xi^{a_{1} a_{2}} \xi^{b_{1} b_{2}} \xi^{-a_{1} a_{2}-a_{1} b_{2}-a_{2} b_{1}-b_{1} b_{2}}=1
\end{aligned}
$$

Thus $\alpha_{1,2}^{\prime}\left(t \rightarrow \alpha_{1,2}^{\prime}\right)=1$ and therefore $\left[\alpha_{1,2}^{\prime}\right]=\left[\alpha_{1,2}\right] \in \operatorname{Im} \Psi$.

Then

- $\operatorname{Im} \Psi=M(G)=\left\langle\left[\alpha_{1,2}^{\prime}\right]\right\rangle$.

- $\hat{G}^{L}=\left\langle\beta_{1} \beta_{2}\right\rangle$ and $N_{t}(\hat{G})=\left\langle\beta_{1} \beta_{2}\right\rangle$.

- $\operatorname{Ker} \Psi=\{1\}$.

Define $\left(\tau_{p, q}^{\prime}\right)^{j}$ via $\left(\left(\tau_{p, q}^{\prime}\right)^{j}\right)_{t}=\left(\alpha_{1,2}^{\prime}\right)^{j}$. Then $\left(\tau_{p, q}^{\prime}\right)^{j}$ is a dual cocycle. Define $\beta \in$ $\left(k^{G}\right)^{\times}$via

$$
\beta\left(x^{a_{1}} y^{a_{2}}\right)=\xi^{a_{1} a_{2}}
$$

Since $\beta(g)=\gamma(g) \gamma(t \rightarrow g)$ for $\gamma \in\left(k^{G}\right)^{\times}$defined in formula (4.5),

$$
\left[\left(\operatorname{triv},\left(\tau_{1,2}^{\prime}\right)^{j}\right)\right]=\left[\left(\sigma^{\beta^{j}}, \tau_{1,2}^{j}\right)\right] \in \mathrm{H}^{2}\left(k L, k^{G}\right)
$$

Thus

$$
\mathrm{H}^{2}\left(k L, k^{G}\right)=\left\langle\left[\left(\operatorname{triv}, \tau_{1,2}^{\prime}\right)\right]\right\rangle=\left\langle\left[\left(\sigma^{\beta}, \tau_{1,2}^{j}\right)\right]\right\rangle \cong\left\langle\left[\alpha_{1,2}^{\prime}\right]\right\rangle \cong \mathbb{Z}_{2^{n}}
$$

4.2. Case 2: $t \rightarrow x=x^{-1}, \quad t \rightarrow y=y^{-1}$.

Proposition 4.5. In this case

$$
\mathrm{H}^{2}\left(k L, k^{G}\right) \cong \operatorname{Ker} \Psi \times \operatorname{Im} \Psi \cong\left\langle\left[\alpha_{1,1}^{2^{n-1}}\right]\right\rangle \times\left\langle\left[\alpha_{2,2}^{2^{n-1}}\right]\right\rangle \times\left\langle\left[\alpha_{1,2}^{2^{n-1}}\right]\right\rangle \cong \mathbb{Z}_{2} \times \mathbb{Z}_{2} \times \mathbb{Z}_{2} .
$$

Proof. Since $t \rightarrow x=x^{-1}$ and $t \rightarrow y=y^{-1}$,

$$
\begin{aligned}
t \rightarrow \alpha_{1,2} & =\alpha_{1,2} \\
t \rightarrow \beta_{j} & =\beta_{j}^{-1} \quad \text { for } j=1,2
\end{aligned}
$$

Thus

- $\operatorname{Im} \Psi=M(G)^{-}=\left\langle\left[\alpha_{1,2}^{2^{n-1}}\right]\right\rangle$.

- $\hat{G}^{L}=\left\langle\beta_{1}^{2^{n-1}}\right\rangle \times\left\langle\beta_{2}^{2^{n-1}}\right\rangle$ and $N_{t}(\hat{G})=\{1\}$.

- $\operatorname{Ker} \Psi=\left\langle\left[\left(\sigma^{\beta_{1}^{2 n-1}}\right.\right.\right.$, triv $\left.\left.)\right]\right\rangle \times\left\langle\left[\left(\sigma^{\beta_{2}^{n-1}}\right.\right.\right.$, triv $\left.\left.)\right]\right\rangle \cong \mathbb{Z}_{2} \times \mathbb{Z}_{2}$.

By parts 3 and 4 of Proposition 4.1, $\left[\left(\sigma^{\beta_{j}^{n-1}}\right.\right.$, triv $\left.)\right]=\left[\left(\operatorname{triv}, \tau_{j, j}^{2^{n-1}}\right)\right]$ for $j=1,2$. Thus

$$
\operatorname{Ker} \Psi=\left\langle\left[\left(\operatorname{triv}, \tau_{1,1}^{2^{n-1}}\right)\right]\right\rangle \times\left\langle\left[\left(\operatorname{triv}, \tau_{2,2}^{2^{n-1}}\right)\right]\right\rangle \cong\left\langle\left[\alpha_{1,1}^{2^{n-1}}\right]\right\rangle \times\left\langle\left[\alpha_{2,2}^{2^{n-1}}\right]\right\rangle
$$

and

$$
\mathrm{H}^{2}\left(k L, k^{G}\right) \cong \mathrm{H}_{c}^{2}\left(k L, k^{G}\right) \cong\left\langle\left[\alpha_{1,1}^{2^{n-1}}\right]\right\rangle \times\left\langle\left[\alpha_{1,2}^{2^{n-1}}\right]\right\rangle \times\left\langle\left[\alpha_{2,2}^{2^{n-1}}\right]\right\rangle \cong \mathbb{Z}_{2} \times \mathbb{Z}_{2} \times \mathbb{Z}_{2}
$$


4.3. Case 3: $t \rightarrow x=x^{1+2^{n-1}}, \quad t \rightarrow y=y^{1+2^{n-1}}$.

In this case we assume that $n \geq 3$, since for $n=2$ the matrix corresponding to the action of $t$ is equal to the matrix from Case 2. Then

Proposition 4.6. In this case

$$
\mathrm{H}^{2}\left(k L, k^{G}\right) \cong \operatorname{Im} \Psi \cong\left\langle\left[\alpha_{1,2}^{2^{n-1}}\right]\right\rangle \cong \mathbb{Z}_{2}
$$

Proof. Since $t \rightarrow x=x^{1+2^{n-1}}$ and $t \rightarrow y=y^{1+2^{n-1}}$,

$$
\begin{aligned}
t \rightarrow \alpha_{1,2} & =\alpha_{1,2} \\
t \rightarrow \beta_{j} & =\beta_{j}^{1+2^{n-1}} \quad \text { for } j=1,2
\end{aligned}
$$

Thus

- $\operatorname{Im} \Psi=M(G)^{-}=\left\langle\left[\alpha_{1,2}^{2^{n-1}}\right]\right\rangle$.

- $\hat{G}^{L}=\left\langle\beta_{1}^{2}\right\rangle \times\left\langle\beta_{2}^{2}\right\rangle$ and $N_{t}(\hat{G})=\left\langle\beta_{1}^{2}\right\rangle \times\left\langle\beta_{2}^{2}\right\rangle$.

- $\operatorname{Ker} \Psi=\{1\}$.

Therefore

$$
\mathrm{H}^{2}\left(k L, k^{G}\right) \cong \mathrm{H}_{c}^{2}\left(k L, k^{G}\right) \cong\left\langle\left[\alpha_{1,2}^{2^{n-1}}\right]\right\rangle \cong \mathbb{Z}_{2}
$$

4.4. Case 4: $t \rightarrow x=x^{-1+2^{n-1}}, \quad t \rightarrow y=y^{-1+2^{n-1}}$.

In this case we assume that $n \geq 3$, since for $n=2$ this action is trivial.

Proposition 4.7. In this case

$$
\mathrm{H}^{2}\left(k L, k^{G}\right) \cong \operatorname{Im} \Psi \cong\left\langle\left[\alpha_{1,2}^{2^{n-1}}\right]\right\rangle \cong \mathbb{Z}_{2}
$$

Proof. Since $t \rightarrow x=x^{-1+2^{n-1}}$ and $t \rightarrow y=y^{-1+2^{n-1}}$,

$$
\begin{aligned}
t \rightarrow \alpha_{1,2} & =\alpha_{1,2} \\
t \rightarrow \beta_{j} & =\beta_{j}^{-1+2^{n-1}} \quad \text { for } j=1,2
\end{aligned}
$$

Thus

- $\operatorname{Im} \Psi=M(G)^{-}=\left\langle\left[\alpha_{1,2}^{2^{n-1}}\right]\right\rangle$.

- $\hat{G}^{L}=\left\langle\beta_{1}^{2^{n-1}}\right\rangle \times\left\langle\beta_{2}^{2^{n-1}}\right\rangle$ and $N_{t}(\hat{G})=\left\langle\beta_{1}^{2^{n-1}}\right\rangle \times\left\langle\beta_{2}^{2^{n-1}}\right\rangle$.

- $\operatorname{Ker} \Psi=\{1\}$.

Therefore

$$
\mathrm{H}^{2}\left(k L, k^{G}\right) \cong \mathrm{H}_{c}^{2}\left(k L, k^{G}\right) \cong\left\langle\left[\alpha_{1,2}^{2^{n-1}}\right]\right\rangle \cong \mathbb{Z}_{2}
$$


4.5. Case 5: $t \rightarrow x=x, \quad t \rightarrow y=y^{1+2^{n-1}}$.

In this case we assume that $n \geq 3$, since for $n=2$ the matrix corresponding to the action of $t$ is equal to the matrix from Case 7 .

Proposition 4.8. In this case

$\mathrm{H}^{2}\left(k L, k^{G}\right)=\left\langle\left[\left(\sigma^{\beta_{1}}\right.\right.\right.$, triv $\left.\left.)\right]\right\rangle \times\left\langle\left[\left(\operatorname{triv}, \tau_{1,2}^{2^{n-1}}\right)\right]\right\rangle \cong \operatorname{Ker} \Psi \times \operatorname{Im} \Psi \cong\left\langle\left[\alpha^{\prime}\right]\right\rangle \times\left\langle\left[\alpha_{1,2}^{2^{n-1}}\right]\right\rangle \cong \mathbb{Z}_{2} \times \mathbb{Z}_{2}$ for $\alpha^{\prime}$ defined via

$$
\alpha^{\prime}\left(x^{a_{1}} y^{a_{2}}, x^{b_{1}} y^{b_{2}}\right)=(-1)^{k}
$$

where $k=0$ if $0 \leq a_{1}+b_{1}<2^{n}$ and $k=1$ if $2^{n} \leq a_{1}+b_{1}<2^{n+1}$.

Proof. Since $t \rightarrow x=x$ and $t \rightarrow y=y^{1+2^{n-1}}$,

$$
\begin{aligned}
t \rightarrow \alpha_{1,2} & =\alpha_{1,2}^{1+2^{n-1}} \\
t \rightarrow \beta_{1} & =\beta_{1} \\
t \rightarrow \beta_{2} & =\beta_{2}^{1+2^{n-1}}
\end{aligned}
$$

Thus

- $\operatorname{Im} \Psi=M(G)^{-}=\left\langle\left[\alpha_{1,2}^{2^{n-1}}\right]\right\rangle$.

- $\hat{G}^{L}=\left\langle\beta_{1}\right\rangle \times\left\langle\beta_{2}^{2}\right\rangle$ and $N_{t}(\hat{G})=\left\langle\beta_{1}^{2}\right\rangle \times\left\langle\beta_{2}^{2}\right\rangle$.

- $\operatorname{Ker} \Psi=\left\langle\left[\left(\sigma^{\beta_{1}}\right.\right.\right.$, triv $\left.\left.)\right]\right\rangle \cong \mathbb{Z}_{2}$.

By part 2 of Proposition $4.1,\left[\left(\sigma^{\beta_{1}}\right.\right.$, triv $\left.)\right]=\left[\left(\operatorname{triv}, \tau^{\prime}\right)\right]$ where $\tau_{t}^{\prime}=\alpha^{\prime}$. Therefore $\mathrm{H}^{2}\left(k L, k^{G}\right)=\left\langle\left[\left(\sigma^{\beta_{1}}\right.\right.\right.$, triv $\left.\left.)\right]\right\rangle \times\left\langle\left[\left(\operatorname{triv}, \tau_{1,2}^{2^{n-1}}\right)\right]\right\rangle \cong\left\langle\left[\alpha^{\prime}\right]\right\rangle \times\left\langle\left[\alpha_{1,2}^{2^{n-1}}\right]\right\rangle \cong \mathbb{Z}_{2} \times \mathbb{Z}_{2}$.

4.6. Case 6: $t \rightarrow x=x^{-1}, \quad t \rightarrow y=y^{-1+2^{n-1}}$.

In this case we assume that $n \geq 3$, since for $n=2$ the matrix corresponding to the action of $t$ is similar to the matrix from Case 7 .

Proposition 4.9. In this case

$$
\mathrm{H}^{2}\left(k L, k^{G}\right) \cong \operatorname{Ker} \Psi \times \operatorname{Im} \Psi \cong\left\langle\alpha_{1,1}^{2^{n-1}}\right\rangle \times\left\langle\alpha_{1,2}^{2^{n-1}}\right\rangle \cong \mathbb{Z}_{2} \times \mathbb{Z}_{2}
$$

Proof. Since $t \rightarrow x=x^{-1}$ and $t \rightarrow y=y^{-1+2^{n-1}}$,

$$
\begin{aligned}
t \rightarrow \alpha_{1,2} & =\alpha_{1,2}^{1+2^{n-1}} \\
t \rightarrow \beta_{1} & =\beta_{1}^{-1} \\
t \rightarrow \beta_{2} & =\beta_{2}^{-1+2^{n-1}}
\end{aligned}
$$

Thus

- $\operatorname{Im} \Psi=M(G)^{-}=\left\langle\left[\alpha_{1,2}^{2^{n-1}}\right]\right\rangle$. 
- $\hat{G}^{L}=\left\langle\beta_{1}^{2^{n-1}}\right\rangle \times\left\langle\beta_{2}^{2^{n-1}}\right\rangle$ and $N_{t}(\hat{G})=\left\langle\beta_{2}^{2^{n-1}}\right\rangle$.

- $\operatorname{Ker} \Psi=\left\langle\left[\left(\sigma^{\beta_{1}^{n-1}}\right.\right.\right.$, triv $\left.\left.)\right]\right\rangle \cong \mathbb{Z}_{2}$.

By part 4 of Proposition 4.1, $\left[\left(\sigma^{\beta_{1}^{2^{n-1}}}, \operatorname{triv}\right)\right]=\left[\left(\operatorname{triv}, \tau_{1,1}^{2^{n-1}}\right)\right]$. Thus

$$
\operatorname{Ker} \Psi=\left\langle\left[\left(\operatorname{triv}, \tau_{1,1}^{2^{n-1}}\right)\right]\right\rangle \cong\left\langle\alpha_{1,1}^{2^{n-1}}\right\rangle
$$

and

$$
\mathrm{H}^{2}\left(k L, k^{G}\right) \cong \mathrm{H}_{c}^{2}\left(k L, k^{G}\right) \cong\left\langle\alpha_{1,1}^{2^{n-1}}\right\rangle \times\left\langle\alpha_{1,2}^{2^{n-1}}\right\rangle \cong \mathbb{Z}_{2} \times \mathbb{Z}_{2}
$$

4.7. Case 7: $t \rightarrow x=x \quad t \rightarrow y=y^{-1}$.

Proposition 4.10. In this case

$$
\begin{aligned}
\mathrm{H}^{2}\left(k L, k^{G}\right) & =\left\langle\left[\left(\sigma^{\beta_{1}}, \operatorname{triv}\right)\right]\right\rangle \times\left\langle\left[\left(\sigma^{\beta_{2}^{2^{n-1}}}, \operatorname{triv}\right)\right]\right\rangle \times\left\langle\left[\left(\operatorname{triv}, \tau_{1,2}\right)\right]\right\rangle \cong \operatorname{Ker} \Psi \times \operatorname{Im} \Psi \\
& \cong\left\langle\alpha^{\prime}\right\rangle \times\left\langle\alpha_{2,2}^{2^{n-1}}\right\rangle \times\left\langle\alpha_{1,2}\right\rangle \cong \mathbb{Z}_{2} \times \mathbb{Z}_{2} \times \mathbb{Z}_{2^{n}}
\end{aligned}
$$

for $\alpha^{\prime}$ defined via

$$
\alpha^{\prime}\left(x^{a_{1}} y^{a_{2}}, x^{b_{1}} y^{b_{2}}\right)=(-1)^{k}
$$

where $k=0$ if $0 \leq a_{1}+b_{1}<2^{n}$ and $k=1$ if $2^{n} \leq a_{1}+b_{1}<2^{n+1}$.

Proof. Since $t \rightarrow x=x$ and $t \rightarrow y=y^{-1}$,

$$
\begin{aligned}
t \rightarrow \alpha_{1,2} & =\alpha_{1,2}^{-1} \\
t \rightarrow \beta_{1} & =\beta_{1} \\
t \rightarrow \beta_{2} & =\beta_{2}^{-1}
\end{aligned}
$$

Thus

- $\operatorname{Im} \Psi=M(G)$.

- $\hat{G}^{L}=\left\langle\beta_{1}\right\rangle \times\left\langle\beta_{2}^{2^{n-1}}\right\rangle$ and $N_{t}(\hat{G})=\left\langle\beta_{1}^{2}\right\rangle$.

- $\operatorname{Ker} \Psi=\left\langle\left[\left(\sigma^{\beta_{1}}\right.\right.\right.$, triv $\left.\left.)\right]\right\rangle \times\left\langle\left[\left(\sigma^{\beta_{2}^{2 n-1}}\right.\right.\right.$, triv $\left.\left.)\right]\right\rangle \cong \mathbb{Z}_{2} \times \mathbb{Z}_{2}$.

- $\mathrm{H}^{2}\left(k L, k^{G}\right)=\left\langle\left[\left(\sigma^{\beta_{1}}, \operatorname{triv}\right)\right]\right\rangle \times\left\langle\left[\left(\sigma^{\beta_{2}^{n-1}}, \operatorname{triv}\right)\right]\right\rangle \times\left\langle\left[\left(\operatorname{triv}, \tau_{1,2}\right)\right]\right\rangle$.

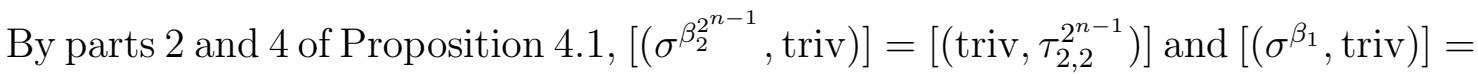
$\left[\left(\operatorname{triv}, \tau^{\prime}\right)\right]$ where $\tau_{t}^{\prime}=\alpha^{\prime}$. Therefore

$$
\mathrm{H}^{2}\left(k L, k^{G}\right) \cong \mathrm{H}_{c}^{2}\left(k L, k^{G}\right) \cong\left\langle\alpha^{\prime}\right\rangle \times\left\langle\alpha_{2,2}^{2^{n-1}}\right\rangle \times\left\langle\alpha_{1,2}\right\rangle \cong \mathbb{Z}_{2} \times \mathbb{Z}_{2} \times \mathbb{Z}_{2^{n}}
$$


4.8. Case 8: $t \rightarrow x=x^{1+2^{n-1}} \quad t \rightarrow y=y^{-1+2^{n-1}}$.

In this case we assume that $n \geq 3$, since for $n=2$ the matrix corresponding to the action of $t$ is similar to the matrix from Case 7 .

Proposition 4.11. In this case

$$
\mathrm{H}^{2}\left(k L, k^{G}\right) \cong \operatorname{Im} \Psi \cong\left\langle\alpha_{1,2}\right\rangle \cong \mathbb{Z}_{2}^{2^{n}}
$$

Proof. Since $t \rightarrow x=x^{1+2^{n-1}}$ and $t \rightarrow y=y^{-1+2^{n-1}}$,

$$
\begin{aligned}
t \rightarrow \alpha_{1,2} & =\alpha_{1,2}^{-1} \\
t \rightarrow \beta_{1} & =\beta_{1}^{1+2^{n-1}} \\
t \rightarrow \beta_{2} & =\beta_{2}^{-1+2^{n-1}}
\end{aligned}
$$

Thus

- $\operatorname{Im} \Psi=M(G)$.

- $\hat{G}^{L}=\left\langle\beta_{1}^{2}\right\rangle \times\left\langle\beta_{2}^{2^{n-1}}\right\rangle$ and $N_{t}(\hat{G})=\left\langle\beta_{1}^{2}\right\rangle \times\left\langle\beta_{2}^{2^{n-1}}\right\rangle$.

- $\operatorname{Ker} \Psi=\{1\}$.

Therefore

$$
\mathrm{H}^{2}\left(k L, k^{G}\right) \cong \mathrm{H}_{c}^{2}\left(k L, k^{G}\right) \cong\left\langle\alpha_{1,2}\right\rangle \cong \mathbb{Z}_{2}^{2^{n}}
$$

4.9. Case 9: $t \rightarrow x=x \quad t \rightarrow y=y^{-1+2^{n-1}}$.

In this case we assume that $n \geq 3$, since for $n=2$ this action is trivial.

Proposition 4.12. In this case

$\mathrm{H}^{2}\left(k L, k^{G}\right)=\left\langle\left[\left(\sigma^{\beta_{1}}\right.\right.\right.$, triv $\left.\left.)\right]\right\rangle \times\left\langle\left[\left(\operatorname{triv}, \tau_{1,2}^{2}\right)\right]\right\rangle \cong \operatorname{Ker} \Psi \times \operatorname{Im} \Psi \cong\left\langle\alpha^{\prime}\right\rangle \times\left\langle\alpha_{1,2}^{2}\right\rangle \cong \mathbb{Z}_{2} \times \mathbb{Z}_{2^{n-1}}$ for $\alpha^{\prime}$ defined via

$$
\alpha^{\prime}\left(x^{a_{1}} y^{a_{2}}, x^{b_{1}} y^{b_{2}}\right)=(-1)^{k}
$$

where $k=0$ if $0 \leq a_{1}+b_{1}<2^{n}$ and $k=1$ if $2^{n} \leq a_{1}+b_{1}<2^{n+1}$.

Proof. Since $t \rightarrow x=x$ and $t \rightarrow y=y^{-1+2^{n-1}}$,

$$
\begin{aligned}
t \rightarrow \alpha_{1,2} & =\alpha_{1,2}^{-1+2^{n-1}} \\
t \rightarrow \beta_{1} & =\beta_{1}^{1+2^{n-1}} \\
t \rightarrow \beta_{2} & =\beta_{2}^{-1+2^{n-1}}
\end{aligned}
$$

Thus

- $\operatorname{Im} \Psi=M(G)^{-}=\left\langle\left[\alpha_{1,2}\right]^{2}\right\rangle$.

- $\hat{G}^{L}=\left\langle\beta_{1}\right\rangle \times\left\langle\beta_{2}^{2^{n-1}}\right\rangle$ and $N_{t}(\hat{G})=\left\langle\beta_{1}^{2}\right\rangle \times\left\langle\beta_{2}^{2^{n-1}}\right\rangle$.

- $\operatorname{Ker} \Psi=\left\langle\left[\left(\sigma^{\beta_{1}}\right.\right.\right.$, triv $\left.\left.)\right]\right\rangle \cong \mathbb{Z}_{2}$. 
By part 2 of Proposition 4.1 , $\left[\left(\sigma^{\beta_{1}}, \operatorname{triv}\right)\right]=\left[\left(\operatorname{triv}, \tau^{\prime}\right)\right]$ where $\tau_{t}^{\prime}=\alpha^{\prime}$. Therefore

$$
\mathrm{H}^{2}\left(k L, k^{G}\right)=\left\langle\left[\left(\sigma^{\beta_{1}}, \text { triv }\right)\right]\right\rangle \times\left\langle\left[\left(\operatorname{triv}, \tau_{1,2}^{2}\right)\right]\right\rangle \cong\left\langle\alpha^{\prime}\right\rangle \times\left\langle\alpha_{1,2}^{2}\right\rangle \cong \mathbb{Z}_{2} \times \mathbb{Z}_{2^{n-1}} .
$$

4.10. Case 10: $t \rightarrow x=x^{1+2^{n-1}} \quad t \rightarrow y=y^{-1}$.

In this case we assume that $n \geq 3$, since for $n=2$ the matrix corresponding to the action of $t$ is equal to the matrix from Case 2. Then

Proposition 4.13. In this case

$$
\mathrm{H}^{2}\left(k L, k^{G}\right) \cong \operatorname{Ker} \Psi \times \operatorname{Im} \Psi \cong\left\langle\alpha_{2,2}^{2^{n-1}}\right\rangle \times\left\langle\alpha_{1,2}^{2}\right\rangle \cong \mathbb{Z}_{2} \times \mathbb{Z}_{2}^{2^{n-1}}
$$

Proof. Since $t \rightarrow x=x^{1+2^{n-1}}$ and $t \rightarrow y=y^{-1}$,

$$
\begin{aligned}
t \rightarrow \alpha_{1,2} & =\alpha_{1,2}^{-1+2^{n-1}} \\
t \rightarrow \beta_{1} & =\beta_{1}^{1+2^{n-1}} \\
t \rightarrow \beta_{2} & =\beta_{2}^{-1}
\end{aligned}
$$

Thus

- $\operatorname{Im} \Psi=M(G)^{-}=\left\langle\left[\alpha_{1,2}\right]^{2}\right\rangle$.

- $\hat{G}^{L}=\left\langle\beta_{1}^{2}\right\rangle \times\left\langle\beta_{2}^{2^{n-1}}\right\rangle$ and $N_{t}(\hat{G})=\left\langle\beta_{1}^{2}\right\rangle$.

- $\operatorname{Ker} \Psi=\left\langle\left[\left(\sigma^{\beta_{2}^{n-1}}\right.\right.\right.$, triv $\left.\left.)\right]\right\rangle \cong \mathbb{Z}_{2}$.

By part 4 of Proposition 4.1, $\left[\left({\sigma^{2_{2}^{n-1}}}^{\text {triv })}\right]=\left[\left(\operatorname{triv}, \tau_{2,2}^{2^{n-1}}\right)\right]\right.$. Thus

$$
\operatorname{Ker} \Psi=\left\langle\left[\left(\operatorname{triv}, \tau_{2,2}^{2^{n-1}}\right)\right]\right\rangle \cong\left\langle\alpha_{2,2}^{2^{n-1}}\right\rangle
$$

and

$$
\mathrm{H}^{2}\left(k L, k^{G}\right) \cong \mathrm{H}_{c}^{2}\left(k L, k^{G}\right) \cong\left\langle\alpha_{2,2}^{2^{n-1}}\right\rangle \times\left\langle\alpha_{1,2}^{2}\right\rangle \cong \mathbb{Z}_{2} \times \mathbb{Z}_{2}^{2^{n-1}}
$$

4.11. Case 11: $t \rightarrow x=x y^{2^{n-1}}, \quad t \rightarrow y=y$.

Proposition 4.14. In this case

$\mathrm{H}^{2}\left(k L, k^{G}\right)=\left\langle\left[\left(\sigma^{\beta_{1}}\right.\right.\right.$, triv $\left.\left.)\right]\right\rangle \times\left\langle\left[\left(\operatorname{triv}, \tau_{1,2}^{2}\right)\right]\right\rangle \cong \operatorname{Ker} \Psi \times \operatorname{Im} \Psi \cong\left\langle\alpha^{\prime}\right\rangle \times\left\langle\alpha_{1,2}^{2^{n-1}}\right\rangle \cong \mathbb{Z}_{2} \times \mathbb{Z}_{2^{n-1}}$ for $\alpha^{\prime}$ defined via

$$
\alpha^{\prime}\left(x^{a_{1}} y^{a_{2}}, x^{b_{1}} y^{b_{2}}\right)=(-1)^{k}
$$

where $k=0$ if $0 \leq a_{1}+b_{1}<2^{n}$ and $k=1$ if $2^{n} \leq a_{1}+b_{1}<2^{n+1}$. 
Proof. Since $t \rightarrow x=x y^{2^{n-1}}$ and $t \rightarrow y=y$,

$$
\begin{aligned}
t \rightarrow \alpha_{1,2} & =\alpha_{1,1}^{2^{n-1}} \alpha_{1,2} \\
t \rightarrow \beta_{1} & =\beta_{1} \\
t \rightarrow \beta_{2} & =\beta_{1}^{2^{n-1}} \beta_{2}
\end{aligned}
$$

Thus

- $\operatorname{Im} \Psi=M(G)^{-}=\left\langle\left[\alpha_{1,2}^{2^{n-1}}\right]\right\rangle$.

- $\hat{G}^{L}=\left\langle\beta_{1}\right\rangle \times\left\langle\beta_{2}^{2}\right\rangle$ and $N_{t}(\hat{G})=\left\langle\beta_{1}^{2}\right\rangle \times\left\langle\beta_{2}^{2}\right\rangle$.

- $\operatorname{Ker} \Psi=\left\langle\left[\left(\sigma^{\beta_{1}}\right.\right.\right.$, triv $\left.\left.)\right]\right\rangle \cong \mathbb{Z}_{2}$.

By part 2 of Proposition 4.1 , $\left[\left(\sigma^{\beta_{1}}\right.\right.$, triv $\left.)\right]=\left[\left(\operatorname{triv}, \tau^{\prime}\right)\right]$ where $\tau_{t}^{\prime}=\alpha^{\prime}$. Therefore $\mathrm{H}^{2}\left(k L, k^{G}\right)=\left\langle\left[\left(\sigma^{\beta_{1}}\right.\right.\right.$, triv $\left.\left.)\right]\right\rangle \times\left\langle\left[\left(\operatorname{triv}, \tau_{1,2}^{2^{n-1}}\right)\right]\right\rangle \cong\left\langle\alpha^{\prime}\right\rangle \times\left\langle\alpha_{1,2}^{2^{n-1}}\right\rangle \cong \mathbb{Z}_{2} \times \mathbb{Z}_{2}$.

4.12. Case 12: $t \rightarrow x=x^{-1} y^{2^{n-1}}, \quad t \rightarrow y=y^{-1}$.

Proposition 4.15. In this case

$$
\mathrm{H}^{2}\left(k L, k^{G}\right) \cong \operatorname{Ker} \Psi \times \operatorname{Im} \Psi \cong\left\langle\alpha_{1,2}^{2^{n-1}}\right\rangle \times\left\langle\alpha_{2,2}^{2^{n-1}}\right\rangle \cong \mathbb{Z}_{2} \times \mathbb{Z}_{2}
$$

Proof. Since $t \rightarrow x=x^{-1} y^{2^{n-1}}$ and $t \rightarrow y=y^{-1}$,

$$
\begin{aligned}
t \rightarrow \alpha_{1,2} & =\alpha_{1,1}^{2^{n-1}} \alpha_{1,2} \\
t \rightarrow \beta_{1} & =\beta_{1}^{-1} \\
t \rightarrow \beta_{2} & =\beta_{1}^{2^{n-1}} \beta_{2}^{-1}
\end{aligned}
$$

Thus

- $\operatorname{Im} \Psi=M(G)^{-}=\left\langle\left[\alpha_{1,2}^{2^{n-1}}\right]\right\rangle$.

- $\hat{G}^{L}=\left\langle\beta_{1}^{2^{n-1}}\right\rangle \times\left\langle\beta_{2}^{2^{n-1}}\right\rangle$ and $N_{t}(\hat{G})=\left\langle\beta_{1}^{2^{n-1}}\right\rangle$.

- $\operatorname{Ker} \Psi=\left\langle\left[\left(\sigma^{\beta_{2}^{2 n-1}}\right.\right.\right.$, triv $\left.\left.)\right]\right\rangle \cong \mathbb{Z}_{2}$.

By part 4 of Proposition 4.1, $\left[\left({\sigma^{\beta_{2}^{n-1}}}^{\text {triv })}\right)=\left[\left(\operatorname{triv}, \tau_{2,2}^{2^{n-1}}\right)\right]\right.$. Thus

$$
\operatorname{Ker} \Psi=\left\langle\left[\left(\operatorname{triv}, \tau_{2,2}^{2^{n-1}}\right)\right]\right\rangle \cong\left\langle\alpha_{2,2}^{2^{n-1}}\right\rangle
$$

and

$$
\mathrm{H}^{2}\left(k L, k^{G}\right) \cong \mathrm{H}_{c}^{2}\left(k L, k^{G}\right) \cong\left\langle\alpha_{2,2}^{2^{n-1}}\right\rangle \times\left\langle\alpha_{1,2}^{2^{n-1}}\right\rangle \cong \mathbb{Z}_{2} \times \mathbb{Z}_{2}
$$


4.13. Case 13: $t \rightarrow x=x^{1+2^{n-1}} y^{2^{n-1}}, \quad t \rightarrow y=y^{1+2^{n-1}}$.

In this case we assume that $n \geq 3$, since for $n=2$ the matrix corresponding to the action of $t$ is equal to the matrix from Case 12 .

Proposition 4.16. In this case

$$
\mathrm{H}^{2}\left(k L, k^{G}\right) \cong \operatorname{Im} \Psi \cong\left\langle\alpha_{1,2}^{2^{n-1}}\right\rangle \cong \mathbb{Z}_{2}
$$

Proof. Since $t \rightarrow x=x^{1+2^{n-1}} y^{2^{n-1}}$ and $t \rightarrow y=y^{1+2^{n-1}}$,

$$
\begin{aligned}
\left(t \rightarrow \alpha_{1,2}\right)\left(x^{a_{1}} y^{a_{2}}, x^{b_{1}} y^{b_{2}}\right) & =\alpha_{1,2}\left(x^{\left(1+2^{n-1}\right) a_{1}} y^{2^{n-1} a_{1}+\left(1+2^{n-1}\right) a_{2}}, x^{\left(1+2^{n-1}\right) b_{1}} y^{2^{n-1} b_{1}+\left(1+2^{n-1}\right) b_{2}}\right) \\
& =\xi^{\left(1+2^{n-1}\right) a_{1}\left(2^{n-1} b_{1}+\left(1+2^{n-1}\right) b_{2}\right)}=\xi^{a_{1}\left(2^{n-1} b_{1}+b_{2}\right)} \\
\left(t \rightarrow \beta_{1}\right)\left(x^{a_{1}} y^{a_{2}}\right) & =\beta_{1}\left(x^{\left(1+2^{n-1}\right) a_{1}} y^{2^{n-1} a_{1}+\left(1+2^{n-1}\right) a_{2}}\right)=\xi^{\left(1+2^{n-1}\right) a_{1}} \\
\left(t \rightarrow \beta_{2}\right)\left(x^{a_{1}} y^{a_{2}}\right) & =\beta_{2}\left(x^{\left(1+2^{n-1}\right) a_{1}} y^{2^{n-1} a_{1}+\left(1+2^{n-1}\right) a_{2}}\right)=\xi^{2^{n-1} a_{1}+\left(1+2^{n-1}\right) a_{2}}
\end{aligned}
$$

Therefore

$$
\begin{aligned}
t \rightarrow \alpha_{1,2} & =\alpha_{1,1}^{2^{n-1}} \alpha_{1,2} \\
t \rightarrow \beta_{1} & =\beta_{1}^{1+2^{n-1}} \\
t \rightarrow \beta_{2} & =\beta_{1}^{2^{n-1}} \beta_{2}^{1+2^{n-1}}
\end{aligned}
$$

Then

- $\operatorname{Im} \Psi=M(G)^{-}=\left\langle\left[\alpha_{1,2}^{2^{n-1}}\right]\right\rangle$.

- $\hat{G}^{L}=\left\langle\beta_{1}^{2}\right\rangle \times\left\langle\beta_{2}^{2}\right\rangle$ and $N_{t}(\hat{G})=\left\langle\beta_{1}^{2}\right\rangle \times\left\langle\beta_{2}^{2}\right\rangle$.

- $\operatorname{Ker} \Psi=\{1\}$.

- $\mathrm{H}^{2}\left(k L, k^{G}\right) \cong \mathrm{H}_{c}^{2}\left(k L, k^{G}\right) \cong\left\langle\alpha_{1,2}^{2^{n-1}}\right\rangle \cong \mathbb{Z}_{2}$.

4.14. Case 14: $t \rightarrow x=x^{-1+2^{n-1}} y^{2^{n-1}}, \quad t \rightarrow y=y^{-1+2^{n-1}}$.

In this case we assume that $n \geq 3$, since for $n=2$ the matrix corresponding to the action of $t$ is equal to the matrix from Case 11 .

Proposition 4.17. In this case

$$
\mathrm{H}^{2}\left(k L, k^{G}\right) \cong \operatorname{Im} \Psi \cong\left\langle\alpha_{1,2}^{2^{n-1}}\right\rangle \cong \mathbb{Z}_{2}
$$

Proof. Since $t \rightarrow x=x^{-1+2^{n-1}} y^{2^{n-1}}$ and $t \rightarrow y=y^{-1+2^{n-1}}$,

$$
\begin{aligned}
\left(t \rightarrow \alpha_{1,2}\right)\left(x^{a_{1}} y^{a_{2}}, x^{b_{1}} y^{b_{2}}\right) & =\alpha_{1,2}\left(x^{\left(-1+2^{n-1}\right) a_{1}} y^{2^{n-1} a_{1}+\left(-1+2^{n-1}\right) a_{2}}, x^{\left(-1+2^{n-1}\right) b_{1}} y^{2^{n-1} b_{1}+\left(-1+2^{n-1}\right) b_{2}}\right) \\
& =\xi^{\left(-1+2^{n-1}\right) a_{1}\left(2^{n-1} b_{1}+\left(-1+2^{n-1}\right) b_{2}\right)}=\xi^{a_{1}\left(2^{n-1} b_{1}+b_{2}\right)}
\end{aligned}
$$




$$
\begin{aligned}
& \left(t \rightarrow \beta_{1}\right)\left(x^{a_{1}} y^{a_{2}}\right)=\beta_{1}\left(x^{\left(-1+2^{n-1}\right) a_{1}} y^{2^{n-1} a_{1}+\left(-1+2^{n-1}\right) a_{2}}\right)=\xi^{\left(-1+2^{n-1}\right) a_{1}} \\
& \left(t \rightarrow \beta_{2}\right)\left(x^{a_{1}} y^{a_{2}}\right)=\beta_{2}\left(x^{\left(-1+2^{n-1}\right) a_{1}} y^{2^{n-1} a_{1}+\left(-1+2^{n-1}\right) a_{2}}\right)=\xi^{2^{n-1} a_{1}+\left(-1+2^{n-1}\right) a_{2}}
\end{aligned}
$$

Therefore

$$
\begin{aligned}
t \rightarrow \alpha_{1,2} & =\alpha_{1,1}^{2^{n-1}} \alpha_{1,2} \\
t \rightarrow \beta_{1} & =\beta_{1}^{-1+2^{n-1}} \\
t \rightarrow \beta_{2} & =\beta_{1}^{2^{n-1}} \beta_{2}^{-1+2^{n-1}}
\end{aligned}
$$

Then

- $\operatorname{Im} \Psi=M(G)^{-}=\left\langle\left[\alpha_{1,2}^{2^{n-1}}\right]\right\rangle$.

- $\hat{G}^{L}=\left\langle\beta_{1}^{2^{n-1}}\right\rangle \times\left\langle\beta_{2}^{2^{n-1}}\right\rangle$ and $N_{t}(\hat{G})=\left\langle\beta_{1}^{2^{n-1}}\right\rangle \times\left\langle\beta_{2}^{2^{n-1}}\right\rangle$.

- $\operatorname{Ker} \Psi=\{1\}$.

- $\mathrm{H}^{2}\left(k L, k^{G}\right) \cong \mathrm{H}_{c}^{2}\left(k L, k^{G}\right) \cong\left\langle\alpha_{1,2}^{2^{n-1}}\right\rangle \cong \mathbb{Z}_{2}$.

4.15. Case 15: $t \rightarrow x=x y^{2^{n-1}}, \quad t \rightarrow y=x^{2^{n-1}} y^{1+2^{n-1}}$.

Proposition 4.18. (1) When $n \geq 3$,

$$
\mathrm{H}^{2}\left(k L, k^{G}\right) \cong \operatorname{Im} \Psi \cong\left\langle\alpha_{1,2}^{2^{n-1}}\right\rangle \cong \mathbb{Z}_{2}
$$

(2) When $n=2$,

$$
\mathrm{H}^{2}\left(k L, k^{G}\right) \cong \operatorname{Im} \Psi \cong\left\langle\alpha_{1,1} \alpha_{1,2} \alpha_{2,2}\right\rangle \cong \mathbb{Z}_{4}
$$

Proof. Since $t \rightarrow x=x y^{2^{n-1}}$ and $t \rightarrow y=x^{2^{n-1}} y^{1+2^{n-1}}$,

$$
\begin{aligned}
\left(t \rightarrow \alpha_{1,2}\right)\left(x^{a_{1}} y^{a_{2}}, x^{b_{1}} y^{b_{2}}\right) & =\alpha_{1,2}\left(x^{a_{1}+2^{n-1} a_{2}} y^{2^{n-1} a_{1}+a_{2}+2^{n-1} a_{2}}, x^{b_{1}+2^{n-1} b_{2}} y^{2^{n-1} b_{1}+b_{2}+2^{n-1} b_{2}}\right) \\
& =\xi^{\left(a_{1}+2^{n-1} a_{2}\right)\left(2^{n-1} b_{1}+b_{2}+2^{n-1} b_{2}\right)}=\xi^{a_{1} b_{2}}(-1)^{a_{1} b_{2}+a_{1} b_{1}+a_{2} b_{2}} \\
\left(t \rightarrow \alpha_{1,1}\right)\left(x^{a_{1}} y^{a_{2}}, x^{b_{1}} y^{b_{2}}\right) & =\alpha_{1,1}\left(x^{a_{1}+2^{n-1} a_{2}} y^{2^{n-1} a_{1}+a_{2}+2^{n-1} a_{2}}, x^{b_{1}+2^{n-1} b_{2}} y^{2^{n-1} b_{1}+b_{2}+2^{n-1} b_{2}}\right) \\
& =\xi^{\left(a_{1}+2^{n-1} a_{2}\right)\left(b_{1}+2^{n-1} b_{2}\right)}=\xi^{a_{1} b_{1}}(-1)^{a_{1} b_{2}+a_{2} b_{1}} \\
\left(t \rightarrow \alpha_{2,2}\right)\left(x^{a_{1}} y^{a_{2}}, x^{b_{1}} y^{b_{2}}\right) & =\alpha_{2,2}\left(x^{a_{1}+2^{n-1} a_{2}} y^{2^{n-1} a_{1}+a_{2}+2^{n-1} a_{2}}, x^{b_{1}+2^{n-1} b_{2}} y^{2^{n-1}} b_{1}+b_{2}+2^{n-1} b_{2}\right) \\
& =\xi^{\left(2^{n-1} a_{1}+a_{2}+2^{n-1} a_{2}\right)\left(2^{n-1} b_{1}+b_{2}+2^{n-1} b_{2}\right)}=\xi^{a_{2} b_{2}}(-1)^{a_{1} b_{2}+a_{2} b_{1}} \\
\left(t \rightarrow \beta_{1}\right)\left(x^{a_{1}} y^{a_{2}}\right) & =\beta_{1}\left(x^{a_{1}+2^{n-1} a_{2}} y^{2^{n-1} a_{1}+a_{2}+2^{n-1} a_{2}}\right)=\xi^{a_{1}+2^{n-1} a_{2}} \\
\left(t \rightarrow \beta_{2}\right)\left(x^{a_{1}} y^{a_{2}}\right) & =\beta_{2}\left(x^{a_{1}+2^{n-1} a_{2}} y^{2^{n-1} a_{1}+a_{2}+2^{n-1} a_{2}}\right)=\xi^{2^{n-1} a_{1}+a_{2}+2^{n-1} a_{2}}
\end{aligned}
$$


Therefore

$$
\begin{aligned}
t \rightarrow \alpha_{1,2} & =\alpha_{1,2}^{1+2^{n-1}} \alpha_{1,1}^{2^{n-1}} \alpha_{2,2}^{2^{n-1}} \\
t \rightarrow \alpha_{j, j} & =\alpha_{j, j} \alpha_{1,2}^{2} \alpha_{2,1}^{2} \text { for } j=1,2 \\
t \rightarrow \beta_{1} & =\beta_{1} \beta_{2}^{2^{n-1}} \\
t \rightarrow \beta_{2} & =\beta_{1}^{2^{n-1}} \beta_{2}^{1+2^{n-1}}
\end{aligned}
$$

Thus

- $\hat{G}^{L}=\left\langle\beta_{1}^{2}\right\rangle \times\left\langle\beta_{2}^{2}\right\rangle$ and $N_{t}(\hat{G})=\left\langle\beta_{1}^{2}\right\rangle \times\left\langle\beta_{2}^{2}\right\rangle$.

- $\operatorname{Ker} \Psi=\{1\}$

(1) Assume first that $n \geq 3$. Then $\operatorname{Im} \Psi=M(G)^{-}=\left\langle\left[\alpha_{1,2}^{2^{n-1}}\right]\right\rangle$ and

$$
\mathrm{H}^{2}\left(k L, k^{G}\right) \cong \mathrm{H}_{c}^{2}\left(k L, k^{G}\right) \cong \operatorname{Im} \Psi \cong\left\langle\alpha_{1,2}^{2^{n-1}}\right\rangle \cong \mathbb{Z}_{2}
$$

(2) Let us now consider the case of $n=2$. We will first show that $\operatorname{Im} \Psi=M(G)$.

By part 1 of Proposition 4.1, $\left[\alpha_{1,2} \alpha_{1,1} \alpha_{2,2}\right]=\left[\alpha_{1,2}\right]$ in $M(G)$. Moreover,

$$
\left(\alpha_{1,2} \alpha_{1,1} \alpha_{2,2}\right)\left(t \rightarrow\left(\alpha_{1,2} \alpha_{1,1} \alpha_{2,2}\right)\right)=\alpha_{1,2} \alpha_{1,1} \alpha_{2,2} \alpha_{1,2}^{-1} \alpha_{1,1}^{2} \alpha_{2,2}^{2} \alpha_{1,1} \alpha_{1,2}^{2} \alpha_{2,1}^{2} \alpha_{2,2} \alpha_{1,2}^{2} \alpha_{2,1}^{2}=1
$$

Therefore $\left[\alpha_{1,2}\right]=\left[\alpha_{1,2} \alpha_{1,1} \alpha_{2,2}\right] \in \operatorname{Im} \Psi$ and

$$
\mathrm{H}^{2}\left(k L, k^{G}\right) \cong \mathrm{H}_{c}^{2}\left(k L, k^{G}\right) \cong \operatorname{Im} \Psi \cong\left\langle\alpha_{1,1} \alpha_{1,2} \alpha_{2,2}\right\rangle \cong \mathbb{Z}_{4}
$$

4.16. Case 16: $t \rightarrow x=x^{-1} y^{2^{n-1}}, \quad t \rightarrow y=x^{2^{n-1}} y^{-1+2^{n-1}}$.

In this case we assume that $n \geq 3$, since for $n=2$ the matrix corresponding to the action of $t$ is similar to the matrix from Case 15 .

Proposition 4.19. In this case

$$
\mathrm{H}^{2}\left(k L, k^{G}\right) \cong \operatorname{Im} \Psi \cong\left\langle\alpha_{1,2}^{2^{n-1}}\right\rangle \cong \mathbb{Z}_{2} .
$$

Proof. Since $t \rightarrow x=x^{-1} y^{2^{n-1}}$ and $t \rightarrow y=x^{2^{n-1}} y^{-1+2^{n-1}}$,

$$
\begin{aligned}
\left(t \rightarrow \alpha_{1,2}\right)\left(x^{a_{1}} y^{a_{2}}, x^{b_{1}} y^{b_{2}}\right) & =\alpha_{1,2}\left(x^{-a_{1}+2^{n-1} a_{2}} y^{2^{n-1} a_{1}-a_{2}+2^{n-1} a_{2}}, x^{-b_{1}+2^{n-1} b_{2}} y^{2^{n-1} b_{1}-b_{2}+2^{n-1} b_{2}}\right) \\
& =\xi^{\left(-a_{1}+2^{n-1} a_{2}\right)\left(2^{n-1} b_{1}-b_{2}+2^{n-1} b_{2}\right)}=\xi^{a_{1} b_{2}}(-1)^{a_{1} b_{2}+a_{1} b_{1}+a_{2} b_{2}} \\
\left(t \rightarrow \beta_{1}\right)\left(x^{a_{1}} y^{a_{2}}\right) & =\beta_{1}\left(x^{-a_{1}+2^{n-1} a_{2}} y^{2^{n-1} a_{1}-a_{2}+2^{n-1} a_{2}}\right)=\xi^{-a_{1}+2^{n-1} a_{2}} \\
\left(t \rightarrow \beta_{2}\right)\left(x^{a_{1}} y^{a_{2}}\right) & =\beta_{2}\left(x^{-a_{1}+2^{n-1} a_{2}} y^{2^{n-1} a_{1}-a_{2}+2^{n-1} a_{2}}\right)=\xi^{2^{n-1} a_{1}-a_{2}+2^{n-1} a_{2}}
\end{aligned}
$$

Therefore

$$
\begin{aligned}
t \rightarrow \alpha_{1,2} & =\alpha_{1,2}^{1+2^{n-1}} \alpha_{1,1}^{2^{n-1}} \alpha_{2,2}^{2^{n-1}} \\
t \rightarrow \beta_{1} & =\beta_{1}^{-1} \beta_{2}^{2^{n-1}}
\end{aligned}
$$




$$
t \rightarrow \beta_{2}=\beta_{1}^{2^{n-1}} \beta_{2}^{-1+2^{n-1}}
$$

- Since $n \geq 3, \operatorname{Im} \Psi=M(G)^{-}=\left\langle\left[\alpha_{1,2}^{2^{n-1}}\right]\right\rangle$.

- $\hat{G}^{L}=\left\langle\beta_{1}^{2^{n-1}}\right\rangle \times\left\langle\beta_{2}^{2^{n-1}}\right\rangle$ and $N_{t}(\hat{G})=\left\langle\beta_{1}^{2^{n-1}}\right\rangle \times\left\langle\beta_{2}^{2^{n-1}}\right\rangle$.

- $\operatorname{Ker} \Psi=\{1\}$

- $\mathrm{H}^{2}\left(k L, k^{G}\right) \cong \mathrm{H}_{c}^{2}\left(k L, k^{G}\right) \cong\left\langle\alpha_{1,2}^{2^{n-1}}\right\rangle \cong \mathbb{Z}_{2}$.

\section{Hopf algebras of dimension 32 With $\mathbf{G}(H)=\mathbb{Z}_{4} \times \mathbb{Z}_{4}$}

In this section we describe all possible nontrivial semisimple Hopf algebras of dimension 32 with a group of group-like elements isomorphic to $\mathbb{Z}_{4} \times \mathbb{Z}_{4}$. We also compute their groups of central group-like elements, the groups of group-like elements of their duals, and their irreducible representations.

Let $H$ be a nontrivial semisimple Hopf algebra of dimension 32 whose group of group-like elements isomorphic to $\mathbb{Z}_{4} \times \mathbb{Z}_{4}$. As in Section 4 , $H$ is equivalent to the bicrossed product $K \#_{\sigma}^{\tau} F$ of $K=k^{G}=(k G)^{*}$ where $G=\widehat{\mathbf{G}(H)} \cong \mathbb{Z}_{4} \times \mathbb{Z}_{4}$ and $F=k L=k\langle t\rangle \cong k \mathbb{Z}_{2}$ with an action $\rightarrow: F \otimes K \rightarrow K$, trivial coaction, a cocycle $\sigma: F \otimes F \rightarrow K$ and a dual cocycle $\tau: F \rightarrow K \otimes K$, and the action by $t$ being an order 2 Hopf algebra automorphism of $K$. Write

$$
\begin{aligned}
\sigma(t, t) & =\sum_{g \in G} \sigma_{t}(g) p_{g} \\
\tau(t) & =\sum_{g, h \in G} \tau_{t}(g, h) p_{g} \otimes p_{h}
\end{aligned}
$$

Denote such a Hopf algebra by $H \cong K \#_{\sigma}^{\tau} F$ by $H\left(\rightarrow, \sigma_{t}, \tau_{t}\right)$. When $\sigma$ is trivial, we will denote $H$ by $H\left(\rightarrow, \tau_{t}\right)$. By [Mas1, Theorem 4.4 (1)] (or Remark 3.2), we may assume that every Hopf algebra $H$ under consideration is equivalent to $K \#^{\tau} F$ with the trivial cocycle, but for some computations it would be beneficial to consider the representative of the equivalence class of $H$ with non-trivial cocycle $\sigma$ but with simpler dual cocycle $\tau$ (as it was done in [K1] and [K2]).

Elements $p_{g} \# t^{j}$, where $g \in G, j=0,1$, form the basis of of $H$. However, when $\sigma$ is assumed to be trivial, we will just denote them by $p_{g} t^{j}$.

As in Section 4 we define

$$
\begin{aligned}
\beta_{1}\left(x^{a} y^{b}\right) & =i^{a} \\
\beta_{2}\left(x^{a} y^{b}\right) & =i^{b} \\
\alpha_{p, q}\left(x^{a_{1}} y^{a_{2}}, x^{b_{1}} y^{b_{2}}\right) & =i^{a_{p} b_{q}} \\
\left(\tau_{p, q}\right)_{t} & =\alpha_{p, q}
\end{aligned}
$$


For all of the Hopf algebras which we describe, we are going to compute their irreducible representations and try to distinguish these Hopf algebras based on their Grothendieck ring structure. To do that we will compute the groups of group-like elements of their dual Hopf algebras and the squares of their irreducible representations. If the Hopf algebras cannot be distinguished based on this information, we will compute their Frobenius-Schur indicators.

In [KMM] it was shown that for $H \cong(k G)^{*} \#_{\sigma}^{\tau} k L$ all simple left $H$-modules may be described as induced modules from certain twisted group algebras of the stabilizers $L_{g}=\{y \in L \mid y \rightarrow g=g\}$. This approach follows the general outline of the work on more general crossed products $B=A \#_{\sigma} k L$, for any semisimple algebra $A$, in [MW] and [W]. However in [KMM] we got more explicit information more easily, because of the special nature of our algebra $A=(k G)^{*}$ and of the $L$-action on $A$.

Since $L$ has order 2 , then $H$ has only irreducible representations of degrees 1 and 2 and $\left|\mathbf{G}\left(\mathrm{H}^{*}\right)\right|=2\left|G^{L}\right|$ (see [K3, Proposition 3.7]). In particular, when we assume that $\sigma$ is trivial, $\mathbf{G}\left(\mathrm{H}^{*}\right)=\chi_{g} \chi_{t}^{j} \mid g \in G^{L}$ and $j=0,1$ where

$$
\begin{array}{ll}
\chi_{g}\left(p_{h}\right)=\delta_{g, h} & \chi_{g}(t)=1 \\
\chi_{t}\left(p_{h}\right)=\delta_{1, h} & \chi_{g}(t)=-1
\end{array}
$$

We will use [K2] for an explicit description of irreducible representations of degree 2. By [K2, Proposition 3.4], for $H \cong(k G)^{*} \#^{\tau} k L$ every irreducible representation $\pi$ of degree 2 is induced from some irreducible representation $\psi$ of $(k G)^{*}$. By [K2, Remark 2.5]

$$
\begin{aligned}
\pi\left(p_{g}\right) & =\left(\begin{array}{cc}
\psi\left(p_{g}\right) & 0 \\
0 & \psi\left(p_{t} \rightarrow g\right)
\end{array}\right) \\
\pi(t) & =\left(\begin{array}{ll}
0 & 1 \\
1 & 0
\end{array}\right)
\end{aligned}
$$

This representation is irreducible if and only if $g \notin G^{L}$. Thus $H \cong(k G)^{*} \#^{\tau} k L$ has exactly $\frac{|G|-\left|G^{L}\right|}{2}$ irreducible 2-dimensional representations $\pi_{g, t \rightarrow g}$ (where $g$ ranges over a choice of one element in each two-element $L$-orbit of $G$ ) defined by

$$
\begin{aligned}
\pi_{g, t \rightarrow g}\left(p_{h}\right) & =\left(\begin{array}{cc}
\delta_{g, h} & 0 \\
0 & \delta_{g, t \rightarrow h}
\end{array}\right) \\
\pi_{g, t \rightarrow g}(t) & =\left(\begin{array}{ll}
0 & 1 \\
1 & 0
\end{array}\right)
\end{aligned}
$$

Let $\chi_{g, t \rightarrow g}$ be the character of $\pi_{g, t \rightarrow g}$, a 2-dimensional representation of $H \cong$ $(k G)^{*} \#^{\tau} k L$. Applying [KMM, Corollary 4.12] to compute the second FrobeniusSchur indicator $\nu_{2}\left(\chi_{g, t \rightarrow g}\right)$, we get the following formula: 


$$
\nu_{2}\left(\chi_{g, t \rightarrow g}\right)=\left\{\begin{array}{cl}
1, & \text { if } g^{2}=1 \\
\tau_{t}\left(g, g^{-1}\right), & \text { if } g^{2} \neq 1 \text { and } t \rightarrow g=g^{-1} \\
0 & \text { otherwise }
\end{array}\right.
$$

In Theorem 4.2 we have described all nonsimilar actions of $t$ on $G$ which correspond to $2 \times 2$ matrices of order 2 . In the case of $n=2$ (that is, when $\mathbf{G}(H) \cong \mathbb{Z}_{4} \times \mathbb{Z}_{4}$ ), there are 27 matrices of this type which are partitioned into six conjugacy classes with the following representatives and sizes:

(1) $\left[\begin{array}{ll}-1 & 0 \\ 0 & -1\end{array}\right]$ (of size 1 );
(2) $\left[\begin{array}{ll}1 & 0 \\ 0 & -1\end{array}\right]$ (of size 6$) ;$
(3) $\left[\begin{array}{ll}1 & 0 \\ 2 & 1\end{array}\right]$ (of size 3$) ;$
(4) $\left[\begin{array}{ll}-1 & 0 \\ 2 & -1\end{array}\right]$ (of size 3 );
(5) $\left[\begin{array}{ll}1 & 2 \\ 2 & -1\end{array}\right]$ (of size 2$) ;$
(6) $\left[\begin{array}{ll}0 & 1 \\ 1 & 0\end{array}\right]$ (of size 12 );

Therefore every Hopf algebra under consideration will be described in one of the following six propositions:

Proposition 5.1. Assume that

$$
t \rightarrow_{1} x=x^{-1}, \quad t \rightarrow_{1} y=y^{-1} .
$$

Then $H \cong H\left(-_{1}, \alpha_{1,2}^{2}\right)$ or $H\left(\rightarrow_{1}, \alpha_{1,1}^{2} \alpha_{1,2}^{2} \alpha_{2,2}^{2}\right)$. Moreover

(1) $\mathbf{G}(H) \cap Z(H)=\hat{G}^{L}=\left\langle\beta_{1}^{2}\right\rangle \times\left\langle\beta_{2}^{2}\right\rangle \cong \mathbb{Z}_{2} \times \mathbb{Z}_{2}$.

(2) $\mathbf{G}\left(H^{*}\right)=\langle a\rangle \times\langle b\rangle \times\langle c\rangle \cong \mathbb{Z}_{2} \times \mathbb{Z}_{2} \times \mathbb{Z}_{2}$.

(3) $H$ has 6 two-dimensional representations $\pi_{1}, \ldots, \pi_{6}$ with characters $\chi_{1}, \ldots, \chi_{6}$ such that

$$
\begin{aligned}
& \pi_{1}^{2}=\pi_{2}^{2}=1+c+a+a c \\
& \pi_{3}^{2}=\pi_{4}^{2}=1+c+b+b c \\
& \pi_{5}^{2}=\pi_{6}^{2}=1+c+a b+a b c
\end{aligned}
$$

(4) When $H \cong H\left(\rightarrow_{1}, \alpha_{1,2}^{2}\right)$,

$$
\nu_{2}\left(\chi_{j}\right)=\left\{\begin{aligned}
1, & j=1,2,3,4 \\
-1, & j=5,6
\end{aligned}\right.
$$


(5) When $H \cong H\left(\rightarrow_{1}, \alpha_{1,1}^{2} \alpha_{1,2}^{2} \alpha_{2,2}^{2}\right)$,

$$
\nu_{2}\left(\chi_{j}\right)=-1 \quad \text { for } j=1,2,3,4,5,6
$$

Proof. By Proposition 4.5, up to equivalence, there are eight Hopf algebras of this type. Four of them, $H\left(\rightarrow_{1}, \alpha_{1,2}^{2}\right), H\left(\rightarrow_{1}, \alpha_{1,1}^{2} \alpha_{1,2}^{2}\right), H\left(\rightarrow_{1}, \alpha_{1,2}^{2} \alpha_{2,2}^{2}\right)$, and $H\left(\rightarrow_{1}, \alpha_{1,1}^{2} \alpha_{1,2}^{2} \alpha_{2,2}^{2}\right)$ are non-trivial. Define

$$
\begin{aligned}
f_{1}: G & \rightarrow G \text { via } \\
f_{1}(x) & =x y \\
f_{1}(y) & =y
\end{aligned}
$$

and

$$
\begin{aligned}
f_{2}: G & \rightarrow G \text { via } \\
f_{2}(x) & =x \\
f_{2}(y) & =x y
\end{aligned}
$$

Then $f_{j}\left(t \rightarrow_{1} g\right)=t \rightarrow_{1} f_{j}(g)$ and

$$
\begin{aligned}
& \alpha_{1,2}^{2} \circ\left(f_{1} \times f_{1}\right)\left(x^{a_{1}} y^{a_{2}}, x^{b_{1}} y^{b_{2}}\right)=\alpha_{1,2}^{2}\left(x^{a_{1}} y^{a_{1}+a_{2}}, x^{b_{1}} y^{b_{1}+b_{2}}\right)=(-1)^{a_{1}\left(b_{1}+b_{2}\right)} \\
& \alpha_{1,2}^{2} \circ\left(f_{2} \times f_{2}\right)\left(x^{a_{1}} y^{a_{2}}, x^{b_{1}} y^{b_{2}}\right)=\alpha_{1,2}^{2}\left(x^{a_{1}+a_{2}} y^{a_{2}}, x^{b_{1}+b_{2}} y^{b_{2}}\right)=(-1)^{\left(a_{1}+a_{2}\right) b_{2}}
\end{aligned}
$$

and therefore

$$
\begin{aligned}
& \alpha_{1,2}^{2} \circ\left(f_{1} \times f_{1}\right)=\alpha_{1,1}^{2} \alpha_{1,2}^{2} \\
& \alpha_{1,2}^{2} \circ\left(f_{2} \times f_{2}\right)=\alpha_{1,2}^{2} \alpha_{2,2}^{2}
\end{aligned}
$$

Therefore $H\left(\alpha_{j, j}^{2} \alpha_{1,2}^{2}\right) \cong H\left(\alpha_{1,2}^{2}\right)$ for $j=1,2$.

Let $H \cong H\left(\rightarrow_{1}, \alpha_{1,2}^{2}\right)$ or $H\left(\rightarrow_{1}, \alpha_{1,1}^{2} \alpha_{1,2}^{2} \alpha_{2,2}^{2}\right)$. Then $H$ has 8 one-dimensional characters of the type $\chi_{g} \chi_{t}^{j}$ for $j=0,1$ and $g \in G^{L}=\left\{1, x^{2}, y^{2}, x^{2} y^{2}\right\}$ where

$$
\begin{array}{ll}
\chi_{g}\left(p_{h}\right)=\delta_{g, h} & \chi_{g}(t)=1 \\
\chi_{t}\left(p_{h}\right)=\delta_{1, h} & \chi_{g}(t)=-1
\end{array}
$$

For each one-dimensional character $\chi, \chi^{2}=1$. Set $a=\chi_{x^{2}}, b=\chi_{y^{2}}$ and $c=\chi_{t}$. Then

$$
\mathbf{G}\left(H^{*}\right)=\langle a\rangle \times\langle b\rangle \times\langle c\rangle \cong \mathbb{Z}_{2} \times \mathbb{Z}_{2} \times \mathbb{Z}_{2} .
$$

$H$ has 6 two-dimensional characters $\chi_{g, g^{-1}}=\chi_{g, t \rightarrow_{1} g}$ for $g \in\left\{x, x y^{2}, y, x^{2} y, x y, x y^{-1}\right\}$ where

$$
\chi_{g, g^{-1}}\left(p_{h}\right)=\delta_{g, h}+\delta_{g^{-1}, h} \quad \chi_{g, g^{-1}}\left(p_{h} t\right)=0 .
$$

Then

$$
\begin{aligned}
\chi_{x, x^{-1}}^{2} & =\chi_{x y^{2}, x^{-1} y^{2}}^{2}=1+\chi_{t}+\chi_{x^{2}}+\chi_{x^{2}} \chi_{t} \\
\chi_{y, y^{-1}}^{2} & =\chi_{x^{2} y, x^{2} y^{-1}}^{2}=1+\chi_{t}+\chi_{y^{2}}+\chi_{y^{2}} \chi_{t} \\
\chi_{x y, x^{-1} y^{-1}}^{2} & =\chi_{x y^{-1}, x^{-1} y}^{2}=1+\chi_{t}+\chi_{x^{2} y^{2}}+\chi_{x^{2} y^{2}} \chi_{t}
\end{aligned}
$$


Since $t \rightarrow_{1} g=g^{-1}$, by formula (5.1) we have

$$
\nu_{2}\left(\chi_{g, g^{-1}}\right)=\tau_{t}\left(g, g^{-1}\right) \quad \text { for all } g \in\left\{x, x y^{2}, y, x^{2} y, x y, x y^{-1}\right\}
$$

Therefore, when $\tau_{t}=\alpha_{1,2}^{2}$,

$$
\begin{aligned}
\nu_{2}\left(\chi_{x, x^{-1}}\right)=\nu_{2}\left(\chi_{x y^{2}, x^{-1} y^{2}}\right)=\nu_{2}\left(\chi_{y, y^{-1}}\right)=\nu_{2}\left(\chi_{x^{2} y, x^{2} y^{-1}}\right) & =1 \\
\nu_{2}\left(\chi_{x y, x^{-1} y^{-1}}\right)=\nu_{2}\left(\chi_{x y^{-1}, x^{-1} y}\right) & =-1
\end{aligned}
$$

and when $\tau_{t}=\alpha_{1,1}^{2} \alpha_{1,2}^{2} \alpha_{2,2}^{2}$,

$$
\nu_{2}\left(\chi_{g, g^{-1}}\right)=-1 \quad \text { for all } g \in\left\{x, x y^{2}, y, x^{2} y, x y, x y^{-1}\right\}
$$

Proposition 5.2. Assume that

$$
t \rightarrow_{2} x=x, \quad t \rightarrow_{2} y=y^{-1} .
$$

Then $H \cong H\left(\rightarrow_{2}, \alpha_{1,2}\right), H\left(\rightarrow_{2}, \alpha_{1,2}^{2}\right), H\left(\rightarrow_{2}, \alpha_{1,2}^{2} \alpha_{2,2}^{2}\right), H\left(\rightarrow_{2}, \beta_{1}, \alpha_{1,2}\right), H\left(\rightarrow_{2}, \beta_{1}, \alpha_{1,2} \alpha_{2,2}^{2}\right)$ or $H\left(-_{2}, \beta_{1}, \alpha_{1,2}^{2}\right)$. Moreover

(1) $\mathbf{G}(H) \cap Z(H)=\hat{G}^{L}=\left\langle\beta_{1}\right\rangle \times\left\langle\beta_{2}^{2}\right\rangle \cong \mathbb{Z}_{4} \times \mathbb{Z}_{2}$.

(2) $\left|\mathbf{G}\left(H^{*}\right)\right|=16$ and $H$ has 4 two-dimensional representations $\pi_{1}, \ldots, \pi_{4}$ with characters $\chi_{1}, \ldots, \chi_{4}$ such that

$$
\nu_{2}\left(\chi_{3}\right)=\nu_{2}\left(\chi_{4}\right)=0
$$

(3) When $H \cong H\left(\rightarrow_{2}, \alpha_{1,2}\right), \mathbf{G}\left(H^{*}\right)=\left\langle a, b, c \mid a^{4}=b^{2}=c^{2}=1, b a b=a c\right\rangle \cong G_{6}$ and

$$
\begin{aligned}
\pi_{1}^{2} & =\pi_{2}^{2}=1+c+b+b c \\
\pi_{3}^{2} & =\pi_{4}^{2}=a^{2}+a^{2} c+a^{2} b+a^{2} b c \\
\nu_{2}\left(\chi_{1}\right) & =1 \quad \text { and } \quad \nu_{2}\left(\chi_{2}\right)=-1 .
\end{aligned}
$$

(4) When $H \cong H\left(-_{2}, \alpha_{1,2}^{2}\right), \mathbf{G}\left(H^{*}\right)=\langle a\rangle \times\langle b\rangle \times\langle c\rangle \cong \mathbb{Z}_{4} \times \mathbb{Z}_{2} \times \mathbb{Z}_{2}$ and

$$
\begin{aligned}
\pi_{1}^{2} & =\pi_{2}^{2}=1+c+b+b c \\
\pi_{3}^{2} & =\pi_{4}^{2}=a^{2}+a^{2} c+a^{2} b+a^{2} b c \\
\nu_{2}\left(\chi_{1}\right) & =\nu_{2}\left(\chi_{2}\right)=1 .
\end{aligned}
$$

(5) When $H \cong H\left(\rightarrow_{2}, \alpha_{1,2}^{2} \alpha_{2,2}^{2}\right), \mathbf{G}\left(H^{*}\right)=\langle a\rangle \times\langle b\rangle \times\langle c\rangle \cong \mathbb{Z}_{4} \times \mathbb{Z}_{2} \times \mathbb{Z}_{2}$ and

$$
\begin{aligned}
\pi_{1}^{2} & =\pi_{2}^{2}=1+c+b+b c \\
\pi_{3}^{2} & =\pi_{4}^{2}=a^{2}+a^{2} c+a^{2} b+a^{2} b c \\
\nu_{2}\left(\chi_{1}\right) & =\nu_{2}\left(\chi_{2}\right)=-1 .
\end{aligned}
$$


(6) When $H \cong H\left(-_{2}, \beta_{1}, \alpha_{1,2}\right), \mathbf{G}\left(H^{*}\right)=\left\langle a, b \mid a^{8}=b^{2}=1, b a b=a^{5}\right\rangle \cong G_{1}$ and

$$
\begin{aligned}
\pi_{1}^{2} & =\pi_{2}^{2}=1+a^{4}+b+a^{4} b \\
\pi_{3}^{2} & =\pi_{4}^{2}=a^{2}+a^{6}+a^{2} b+a^{6} b \\
\nu_{2}\left(\chi_{1}\right) & =\nu_{2}\left(\chi_{2}\right)=1 .
\end{aligned}
$$

(7) When $H \cong H\left(\rightarrow_{2}, \beta_{1}, \alpha_{1,2} \alpha_{2,2}^{2}\right), \mathbf{G}\left(H^{*}\right)=\left\langle a, b \mid a^{8}=b^{2}=1, b a b=a^{5}\right\rangle \cong G_{1}$ and

$$
\begin{aligned}
\pi_{1}^{2} & =\pi_{2}^{2}=1+a^{4}+b+a^{4} b \\
\pi_{3}^{2} & =\pi_{4}^{2}=a^{2}+a^{6}+a^{2} b+a^{6} b \\
\nu_{2}\left(\chi_{1}\right) & =\nu_{2}\left(\chi_{2}\right)=-1 .
\end{aligned}
$$

(8) When $H \cong H\left(\rightarrow_{2}, \beta_{1}, \alpha_{1,2}^{2}\right), \mathbf{G}\left(H^{*}\right)=\langle a\rangle \times\langle b\rangle \cong \mathbb{Z}_{8} \times \mathbb{Z}_{2}$ and

$$
\begin{aligned}
\pi_{1}^{2} & =\pi_{2}^{2}=1+a^{4}+b+a^{4} b \\
\pi_{3}^{2} & =\pi_{4}^{2}=a^{2}+a^{6}+a^{2} b+a^{6} b \\
\nu_{2}\left(\chi_{1}\right) & =\nu_{2}\left(\chi_{2}\right)=1 .
\end{aligned}
$$

Proof. By Proposition 4.10, up to equivalence, there are sixteen Hopf algebras of this type. Twelve of them, $H\left(\rightarrow_{2}, \beta_{1}^{j} \alpha_{1,2}^{k} \alpha_{2,2}^{l}\right)$ for $j=0,1, k=1,2,3, l=0,2$, are non-trivial. Define

$$
\begin{aligned}
f_{1}: G & \rightarrow G \text { via } \\
f_{1}(x) & =t \rightarrow x=x \\
f_{1}(y) & =t \rightarrow y=y^{-1}
\end{aligned}
$$

and

$$
\begin{aligned}
f_{2}: G & \rightarrow G \text { via } \\
f_{2}(x) & =x \\
f_{2}(y) & =x^{2} y
\end{aligned}
$$

and

$$
\begin{aligned}
& f_{3}: G \rightarrow G \text { via } \\
& f_{3}(x)=x y^{2} \\
& f_{3}(y)=x^{2} y
\end{aligned}
$$

Then $f_{j}\left(t \overrightarrow{2}_{2} g\right)=t \overrightarrow{ }_{2} f_{j}(g)$ and

$$
\begin{aligned}
\alpha_{1,2} \circ\left(f_{1} \times f_{1}\right)\left(x^{a_{1}} y^{a_{2}}, x^{b_{1}} y^{b_{2}}\right) & =i^{-a_{1} b_{2}} \\
\alpha_{1,2} \circ\left(f_{2} \times f_{2}\right)\left(x^{a_{1}} y^{a_{2}}, x^{b_{1}} y^{b_{2}}\right) & =i^{a_{1} b_{2}}(-1)^{a_{2} b_{2}} \\
\alpha_{1,2}^{2} \circ\left(f_{3} \times f_{3}\right)\left(x^{a_{1}} y^{a_{2}}, x^{b_{1}} y^{b_{2}}\right) & =(-1)^{a_{1} b_{2}} \\
\beta_{1} \circ f_{1}\left(x^{a_{1}} y^{a_{2}}\right) & =i^{a_{1}}
\end{aligned}
$$




$$
\begin{aligned}
\beta_{1} \circ f_{3}\left(x^{a_{1}} y^{a_{2}}\right) & =i^{a_{1}}(-1)^{a_{2}} \\
\alpha_{2,2}^{2} \circ\left(f_{j} \times f_{j}\right)\left(x^{a_{1}} y^{a_{2}}, x^{b_{1}} y^{b_{2}}\right) & =(-1)^{a_{2} b_{2}}
\end{aligned}
$$

and therefore

$$
\begin{aligned}
\alpha_{1,2} \circ\left(f_{1} \times f_{1}\right) & =\alpha_{1,2}^{3} \\
\alpha_{1,2} \circ\left(f_{2} \times f_{2}\right) & =\alpha_{1,2} \alpha_{2,2}^{2} \\
\alpha_{1,2}^{2} \circ\left(f_{3} \times f_{3}\right) & =\alpha_{1,2}^{2} \\
\beta_{1} \circ f_{1} & =\beta_{1} \\
\beta_{1} \circ f_{3} & =\beta_{1} \beta_{2}^{2} \\
\alpha_{2,2}^{2} \circ\left(f_{j} \times f_{j}\right) & =\alpha_{2,2}^{2}
\end{aligned}
$$

Therefore

$$
\begin{aligned}
H\left(\rightarrow_{2}, \beta_{1}^{j}, \alpha_{1,2}^{3} \alpha_{2,2}^{l}\right) & \cong H\left(\rightarrow_{2}, \beta_{1}^{j}, \alpha_{1,2} \alpha_{2,2}^{l}\right) \text { for } j=0,1, l=0,2 \\
H\left(\rightarrow_{2}, \alpha_{1,2} \alpha_{2,2}^{2}\right) & \cong H\left(\rightarrow_{2}, \alpha_{1,2}\right) \\
H\left(\rightarrow_{2}, \beta_{1}, \alpha_{1,2}^{2} \alpha_{2,2}^{2}\right) & \cong H\left(\rightarrow_{2}, \beta_{1}, \alpha_{1,2}^{2}\right)
\end{aligned}
$$

For further computations we will use the fact that, by Proposition $4.10, H\left(\rightarrow_{2}, \beta_{1}, \alpha_{1,2}^{j} \alpha_{2,2}^{l}\right)$ is equivalent to $H\left(\rightarrow_{2}, \alpha^{\prime} \alpha_{1,2}^{j} \alpha_{2,2}^{l}\right)$ for $\alpha^{\prime}$ defined via

$$
\alpha^{\prime}\left(x^{a_{1}} y^{a_{2}}, x^{b_{1}} y^{b_{2}}\right)=(-1)^{k}
$$

where $k=0$ if $0 \leq a_{1}+b_{1}<4$ and $k=1$ if $4 \leq a_{1}+b_{1}<8$.

Let $H$ be a Hopf algebra corresponding to $\rightarrow_{2}$. Then $H$ has 16 one-dimensional characters of the type $\chi_{g} \chi_{t}^{j}$ for $j=0,1$ and $g \in G^{L}=\langle x\rangle \times\left\langle y^{2}\right\rangle$ where

$$
\begin{array}{ll}
\chi_{g}\left(p_{h}\right)=\delta_{g, h} & \chi_{g}(t)=1 \\
\chi_{t}\left(p_{h}\right)=\delta_{1, h} & \chi_{g}(t)=-1
\end{array}
$$

Then

$$
\begin{aligned}
\chi_{y^{2}}^{2} & =\chi_{t}^{2}=\chi_{1}=1 \\
\chi_{x}^{2} & =\chi_{x^{2}} \\
\chi_{t} & \in Z\left(\mathbf{G}\left(H^{*}\right)\right)
\end{aligned}
$$

$H$ has 4 two-dimensional characters $\chi_{g, t \rightarrow_{2} g}$ for $g \in\left\{y, x^{2} y, x y, x^{-1} y\right\}$ where

$$
\chi_{g, t \rightarrow_{2} g}\left(p_{h}\right)=\delta_{g, h}+\delta_{t \rightarrow_{2} g, h} \quad \chi_{g, t \rightarrow_{2} g}\left(p_{h} t\right)=0 .
$$

Then

$$
\begin{aligned}
\chi_{y, y^{-1}}^{2} & =\chi_{x^{2} y, x^{2} y^{-1}}^{2}=1+\chi_{t}+\chi_{y^{2}}+\chi_{y^{2}} \chi_{t} \\
\chi_{x y, x^{-1} y^{-1}}^{2} & =\chi_{x y^{-1}, x^{-1} y}^{2}=\chi_{x^{2}}+\chi_{x^{2}} \chi_{t}+\chi_{x^{2} y^{2}}+\chi_{x^{2} y^{2}} \chi_{t}
\end{aligned}
$$


Then by formula (5.1)

$$
\begin{aligned}
\nu_{2}\left(\chi_{g, t-} g\right. & =\tau_{t}\left(g, g^{-1}\right) \quad \text { for } g \in\left\{y, x^{2} y\right\} \\
\nu_{2}\left(\chi_{x y, x^{-1} y^{-1}}\right) & =\nu_{2}\left(\chi_{x y^{-1}, x^{-1} y}\right)=0
\end{aligned}
$$

(1) Assume that $H \cong H\left(\rightarrow_{2}, \alpha_{1,2}\right)$. Then

$$
\begin{aligned}
\chi_{x}^{4} & =1 \\
\chi_{x} \chi_{y^{2}} & =\chi_{y^{2}} \chi_{x} \chi_{t}
\end{aligned}
$$

Denote $a=\chi_{x}, b=\chi_{y^{2}}, c=\chi_{t}$. Then

$$
\mathbf{G}\left(H^{*}\right)=\left\langle a, b, c \mid a^{4}=b^{2}=c^{2}=1, b a b=a c\right\rangle \cong G_{6} .
$$

In addition,

$$
\begin{aligned}
\nu_{2}\left(\chi_{y, y^{-1}}\right) & =\alpha_{1,2}\left(y, y^{-1}\right)=1 \\
\nu_{2}\left(\chi_{x^{2} y, x^{2} y^{-1}}\right) & =\alpha_{1,2}\left(x^{2} y, x^{2} y^{-1}\right)=-1
\end{aligned}
$$

(2) Assume that $H \cong H\left(\rightarrow_{2}, \alpha_{1,2}^{2}\right)$. Then

$$
\begin{aligned}
\chi_{x}^{4} & =1 \\
\chi_{x} \chi_{y^{2}} & =\chi_{y^{2}} \chi_{x}
\end{aligned}
$$

Denote $a=\chi_{x}, b=\chi_{y^{2}}, c=\chi_{t}$. Then

$$
\mathbf{G}\left(H^{*}\right)=\langle a\rangle \times\langle b\rangle \times\langle c\rangle \cong \mathbb{Z}_{4} \times \mathbb{Z}_{2} \times \mathbb{Z}_{2} .
$$

In addition,

$$
\nu_{2}\left(\chi_{y, y^{-1}}\right)=\nu_{2}\left(\chi_{x^{2} y, x^{2} y^{-1}}\right)=1
$$

(3) Assume that $H \cong H\left(\rightarrow_{2}, \alpha_{1,2}^{2} \alpha_{2,2}^{2}\right)$. Then

$$
\begin{aligned}
\chi_{x}^{4} & =1 \\
\chi_{x} \chi_{y^{2}} & =\chi_{y^{2}} \chi_{x}
\end{aligned}
$$

Denote $a=\chi_{x}, b=\chi_{y^{2}}, c=\chi_{t}$. Then

$$
\mathbf{G}\left(H^{*}\right)=\langle a\rangle \times\langle b\rangle \times\langle c\rangle \cong \mathbb{Z}_{4} \times \mathbb{Z}_{2} \times \mathbb{Z}_{2} .
$$

In addition,

$$
\nu_{2}\left(\chi_{y, y^{-1}}\right)=\nu_{2}\left(\chi_{x^{2} y, x^{2} y^{-1}}\right)=-1
$$

(4) Assume that $H \cong H\left(\rightarrow_{2}, \beta_{1}, \alpha_{1,2}\right) \cong H\left(\rightarrow_{2}, \alpha^{\prime} \alpha_{1,2}\right)$. Then

$$
\begin{aligned}
\chi_{x}^{4} & =\chi_{t} \\
\chi_{x} \chi_{y^{2}} & =\chi_{y^{2}} \chi_{x} \chi_{t}=\chi_{y^{2}} \chi_{x}^{5}
\end{aligned}
$$

Denote $a=\chi_{x}$ and $b=\chi_{y^{2}}$. Then

$$
\mathbf{G}\left(H^{*}\right)=\left\langle a, b \mid a^{8}=b^{2}=1, b a b=a^{5}\right\rangle \cong G_{1} .
$$

In addition,

$$
\nu_{2}\left(\chi_{y, y^{-1}}\right)=\nu_{2}\left(\chi_{x^{2} y, x^{2} y^{-1}}\right)=1
$$


(5) Assume that $H \cong H\left(\rightarrow_{2}, \beta_{1}, \alpha_{1,2} \alpha_{2,2}^{2}\right) \cong H\left(\rightarrow_{2}, \alpha^{\prime} \alpha_{1,2} \alpha_{2,2}^{2}\right)$. Then

$$
\begin{aligned}
\chi_{x}^{4} & =\chi_{t} \\
\chi_{x} \chi_{y^{2}} & =\chi_{y^{2}} \chi_{x} \chi_{t}=\chi_{y^{2}} \chi_{x}^{5}
\end{aligned}
$$

Denote $a=\chi_{x}$ and $b=\chi_{y^{2}}$. Then

$$
\mathbf{G}\left(H^{*}\right)=\left\langle a, b \mid a^{8}=b^{2}=1, b a b=a^{5}\right\rangle \cong G_{1} .
$$

In addition,

$$
\nu_{2}\left(\chi_{y, y^{-1}}\right)=\nu_{2}\left(\chi_{x^{2} y, x^{2} y^{-1}}\right)=-1
$$

(6) Assume that $H \cong H\left(\rightarrow_{2}, \beta_{1}, \alpha_{1,2}^{2}\right) \cong H\left(\rightarrow_{2}, \alpha^{\prime} \alpha_{1,2}^{2}\right)$. Then

$$
\begin{aligned}
\chi_{x}^{4} & =\chi_{t} \\
\chi_{x} \chi_{y^{2}} & =\chi_{y^{2}} \chi_{x}
\end{aligned}
$$

Denote $a=\chi_{x}$ and $b=\chi_{y^{2}}$. Then

$$
\mathbf{G}\left(H^{*}\right)=\langle a\rangle \times\langle b\rangle \cong \mathbb{Z}_{8} \times \mathbb{Z}_{2} .
$$

In addition,

$$
\nu_{2}\left(\chi_{y, y^{-1}}\right)=\nu_{2}\left(\chi_{x^{2} y, x^{2} y^{-1}}\right)=1
$$

Proposition 5.3. Assume that

$$
t \rightarrow_{3} x=x y^{2}, \quad t \rightarrow_{3} y=y .
$$

Then $H \cong H\left(-_{3}, \alpha_{1,2}^{2}\right)$ or $H\left(-_{3}, \beta_{1}, \alpha_{1,2}^{2}\right)$. Moreover

(1) $\mathbf{G}(H) \cap Z(H)=\hat{G}^{L}=\left\langle\beta_{1}\right\rangle \times\left\langle\beta_{2}^{2}\right\rangle \cong \mathbb{Z}_{4} \times \mathbb{Z}_{2}$.

(2) $\mathbf{G}\left(H^{*}\right)$ is an abelian group of order 16 and $H$ has 4 two-dimensional representations $\pi_{1}, \ldots, \pi_{4}$ with characters $\chi_{1}, \ldots, \chi_{4}$ such that

$$
\nu_{2}\left(\chi_{j}\right)=0 \quad \text { for } j=1,2,3,4
$$

(3) When $H \cong H\left(-_{3}, \alpha_{1,2}^{2}\right), \mathbf{G}\left(H^{*}\right)=\langle a\rangle \times\langle b\rangle \times\langle c\rangle \cong \mathbb{Z}_{4} \times \mathbb{Z}_{2} \times \mathbb{Z}_{2}$ and

$$
\pi_{j}^{2}=b+b c+a^{2} b+a^{2} b c \quad \text { for } j=1,2,3,4
$$

(4) When $H \cong H\left(-_{3}, \beta_{1}, \alpha_{1,2}^{2}\right), \mathbf{G}\left(H^{*}\right)=\langle a\rangle \times\langle b\rangle \cong \mathbb{Z}_{4} \times \mathbb{Z}_{4}$ and

$$
\pi_{j}^{2}=b+b^{3}+a^{2} b+a^{2} b^{3} \quad \text { for } j=1,2,3,4
$$

Proof. By Proposition 4.14, up to equivalence, there are four Hopf algebras of this type. Two of them, $H\left(-_{3}, \alpha_{1,2}^{2}\right)$ and $H\left(\rightarrow_{3}, \beta_{1}, \alpha_{1,2}^{2}\right)$, are non-trivial.

For further computations we will use the fact that by Proposition $4.14 H\left(\rightarrow_{3}, \beta_{1}, \alpha_{1,2}^{2}\right)$ is equivalent to $H\left(-_{3}, \alpha^{\prime} \alpha_{1,2}^{2}\right)$ for $\alpha^{\prime}$ defined via

$$
\alpha^{\prime}\left(x^{a_{1}} y^{a_{2}}, x^{b_{1}} y^{b_{2}}\right)=(-1)^{k}
$$

where $k=0$ if $0 \leq a_{1}+b_{1}<4$ and $k=1$ if $4 \leq a_{1}+b_{1}<8$. 
Let $H$ be a Hopf algebra corresponding to $\rightarrow_{3}$. Then $H$ has 16 one-dimensional characters of the type $\chi_{g} \chi_{t}^{j}$ for $j=0,1$ and $g \in G^{L}=\left\langle x^{2}\right\rangle \times\langle y\rangle$ where

$$
\begin{array}{ll}
\chi_{g}\left(p_{h}\right)=\delta_{g, h} & \chi_{g}(t)=1 \\
\chi_{t}\left(p_{h}\right)=\delta_{1, h} & \chi_{g}(t)=-1
\end{array}
$$

Then $\mathbf{G}\left(H^{*}\right)$ is abelian and

$$
\chi_{y}^{4}=\chi_{t}^{2}=\chi_{1}=1
$$

$H$ has 4 two-dimensional characters $\chi_{g, t \rightarrow_{3} g}$ for $g \in\left\{x, x^{-1}, x y, x^{-1} y\right\}$ where

$$
\chi_{g, t \rightarrow} g\left(p_{h}\right)=\delta_{g, h}+\delta_{t \rightarrow} g, h \quad \chi_{g, t \rightarrow} g\left(p_{h} t\right)=0 .
$$

Then for all $g \in\left\{x, x^{-1}, x y, x^{-1} y\right\}$

$$
\begin{aligned}
\chi_{g, t \rightarrow_{3} g}^{2} & =\chi_{x^{2}}+\chi_{x^{2}} \chi_{t}+\chi_{x^{2} y^{2}}+\chi_{x^{2} y^{2}} \chi_{t} \\
\nu_{2}\left(\chi_{g, t \rightarrow_{3} g}\right) & =0
\end{aligned}
$$

If $H \cong H\left(\rightarrow_{3}, \alpha_{1,2}^{2}\right)$ then $\chi_{x^{2}}^{2}=1$ and

$$
\mathbf{G}\left(H^{*}\right)=\langle a\rangle \times\langle b\rangle \times\langle c\rangle \cong \mathbb{Z}_{4} \times \mathbb{Z}_{2} \times \mathbb{Z}_{2}
$$

for $a=\chi_{y}, b=\chi_{x^{2}}$, and $c=\chi_{t}$.

If $H \cong H\left(-_{3}, \beta_{1}, \alpha_{1,2}^{2}\right) \cong H\left(\rightarrow_{3}, \alpha^{\prime} \alpha_{1,2}^{2}\right)$ then $\chi_{x^{2}}^{2}=\chi_{t}$ and

$$
\mathbf{G}\left(H^{*}\right)=\langle a\rangle \times\langle b\rangle \cong \mathbb{Z}_{4} \times \mathbb{Z}_{4}
$$

for $a=\chi_{y}$ and $b=\chi_{x^{2}}$.

Proposition 5.4. Assume that

$$
t \rightarrow_{4} x=x^{-1} y^{2}, \quad t \rightarrow_{4} y=y^{-1} .
$$

Then $H \cong H\left(\rightarrow_{4}, \alpha_{1,2}^{2}\right)$ or $H\left(\rightarrow_{4}, \alpha_{1,2}^{2} \alpha_{2,2}^{2}\right)$. Moreover

(1) $\mathbf{G}(H) \cap Z(H)=\hat{G}^{L}=\left\langle\beta_{1}^{2}\right\rangle \times\left\langle\beta_{2}^{2}\right\rangle \cong \mathbb{Z}_{2} \times \mathbb{Z}_{2}$.

(2) $\mathbf{G}\left(H^{*}\right)=\langle a\rangle \times\langle b\rangle \times\langle c\rangle \cong \mathbb{Z}_{2} \times \mathbb{Z}_{2} \times \mathbb{Z}_{2}$.

(3) $H$ has 6 two-dimensional representations $\pi_{1}, \ldots, \pi_{6}$ with characters $\chi_{1}, \ldots, \chi_{6}$ such that

$$
\begin{aligned}
\pi_{1}^{2} & =\pi_{2}^{2}=1+c+b+b c \\
\pi_{3}^{2} & =\pi_{4}^{2}=a+a c+b+b c \\
\pi_{5}^{2} & =\pi_{6}^{2}=a b+a b c+b+b c \\
\nu_{2}\left(\chi_{j}\right) & =0 \text { for } j=3,4,5,6
\end{aligned}
$$

(4) When $H \cong H\left(\rightarrow_{4}, \alpha_{1,2}^{2}\right)$,

$$
\nu_{2}\left(\chi_{j}\right)=1 \quad \text { for } j=1,2
$$

(5) When $H \cong H\left(\rightarrow_{4}, \alpha_{1,2}^{2} \alpha_{2,2}^{2}\right)$,

$$
\nu_{2}\left(\chi_{j}\right)=-1 \quad \text { for } j=1,2
$$


Proof. By Proposition 4.15, up to equivalence, there are four Hopf algebras of this type. Two of them, $H\left(\vec{\rightarrow}_{4}, \alpha_{1,2}^{2}\right)$ and $H\left(\rightarrow_{4}, \beta_{1}, \alpha_{1,2}^{2}\right)$, are non-trivial.

Let $H$ be a Hopf algebra corresponding to $\rightarrow_{4}$. Then $H$ has 8 one-dimensional characters of the type $\chi_{g} \chi_{t}^{j}$ for $j=0,1$ and $g \in G^{L}=\left\langle x^{2}\right\rangle \times\left\langle y^{2}\right\rangle$ where

$$
\begin{array}{ll}
\chi_{g}\left(p_{h}\right)=\delta_{g, h} & \chi_{g}(t)=1 \\
\chi_{t}\left(p_{h}\right)=\delta_{1, h} & \chi_{g}(t)=-1
\end{array}
$$

For each one-dimensional character $\chi, \chi^{2}=1$. Set $a=\chi_{x^{2}}, b=\chi_{y^{2}}$ and $c=\chi_{t}$. Then

$$
\mathbf{G}\left(H^{*}\right)=\langle a\rangle \times\langle b\rangle \times\langle c\rangle \cong \mathbb{Z}_{2} \times \mathbb{Z}_{2} \times \mathbb{Z}_{2} .
$$

$H$ has 6 two-dimensional characters $\chi_{g, t \rightarrow_{4} g}$ for $g \in\left\{y, x^{2} y, x, x^{-1}, x y, x y^{-1}\right\}$ where

$$
\chi_{g, t \rightarrow} g\left(p_{h}\right)=\delta_{g, h}+\delta_{t \rightarrow_{4} g, h} \quad \chi_{g, t \rightarrow_{4} g}\left(p_{h} t\right)=0 .
$$

Then

$$
\begin{aligned}
\chi_{y, y^{-1}}^{2} & =\chi_{x^{2} y, x^{2} y^{-1}}^{2}=1+\chi_{t}+\chi_{y^{2}}+\chi_{y^{2}} \chi_{t} \\
\chi_{x, x^{-1} y^{2}}^{2} & =\chi_{x^{-1}, x y^{2}}^{2}=\chi_{x^{2}}+\chi_{x^{2}} \chi_{t}+\chi_{y^{2}}+\chi_{y^{2}} \chi_{t} \\
\chi_{x y, x^{-1} y}^{2} & =\chi_{x y^{-1}, x^{-1} y^{-1}}^{2}=\chi_{x^{2} y^{2}}+\chi_{x^{2} y^{2}} \chi_{t}+\chi_{y^{2}}+\chi_{y^{2}} \chi_{t} \\
\nu_{2}\left(\chi_{g, t \rightarrow_{4} g}\right) & =0 \text { for } g \in\left\{x, x^{-1}, x y, x y^{-1}\right\}
\end{aligned}
$$

If $H \cong H\left(\rightarrow_{4}, \alpha_{1,2}^{2}\right)$ then by formula (5.1)

$$
\begin{aligned}
\nu_{2}\left(\chi_{y, y^{-1}}\right) & =\alpha_{1,2}^{2}\left(y, y^{-1}\right)=1 \\
\nu_{2}\left(\chi_{x^{2} y, x^{2} y^{-1}}\right) & =\alpha_{1,2}^{2}\left(x^{2} y, x^{2} y^{-1}\right)=1
\end{aligned}
$$

If $H \cong H\left(\rightarrow_{4}, \alpha_{1,2}^{2} \alpha_{2,2}^{2}\right)$ then by formula (5.1)

$$
\begin{aligned}
\nu_{2}\left(\chi_{y, y^{-1}}\right) & =\alpha_{1,2}^{2}\left(y, y^{-1}\right) \alpha_{2,2}^{2}\left(y, y^{-1}\right)=-1 \\
\nu_{2}\left(\chi_{x^{2} y, x^{2} y^{-1}}\right) & =\alpha_{1,2}^{2}\left(x^{2} y, x^{2} y^{-1}\right) \alpha_{2,2}^{2}\left(x^{2} y, x^{2} y^{-1}\right)=-1
\end{aligned}
$$

Proposition 5.5. Assume that

$$
t \rightarrow_{5} x=y, \quad t \rightarrow_{5} y=x .
$$

Then $H \cong H\left(-_{5}, \alpha_{1,2}^{\prime}\right)$ or $H\left(-_{5},\left(\alpha_{1,2}^{\prime}\right)^{2}\right)$ for $\alpha_{1,2}^{\prime}$ defined via

$$
\alpha_{1,2}^{\prime}\left(x^{a_{1}} y^{a_{2}}, x^{b_{1}} y^{b_{2}}\right)=\omega^{a_{1} b_{2}-a_{2} b_{1}}(-1)^{\left(a_{1}+b_{1}\right) k_{2}+\left(a_{2}+b_{2}\right) k_{1}}
$$

where $\omega$ is a primitive 8 -th root of 1 such that $\omega^{2}=i$, and $k_{j}=0$ if $0 \leq a_{j}+b_{j}<4$ and $k_{j}=1$ if $4 \leq a_{j}+b_{j}<8$. Moreover

(1) $\mathbf{G}(H) \cap Z(H)=\hat{G}^{L}=\left\langle\beta_{1} \beta_{2}\right\rangle \cong \mathbb{Z}_{4}$.

(2) $\mathbf{G}\left(H^{*}\right)=\langle a\rangle \times\langle b\rangle \cong \mathbb{Z}_{4} \times \mathbb{Z}_{2}$. 
(3) $H$ has 6 two-dimensional representations $\pi_{1}, \ldots, \pi_{6}$ with characters $\chi_{1}, \ldots, \chi_{6}$ such that

$$
\begin{aligned}
\pi_{1}^{2} & =\pi_{2}^{2}=a+a b+\pi_{6} \\
\pi_{3}^{2} & =\pi_{4}^{2}=a^{3}+a^{3} b+\pi_{6} \\
\pi_{5}^{2} & =\pi_{6}^{2}=1+b+a^{2}+a^{2} b \\
\nu_{2}\left(\chi_{j}\right) & =0 \quad \text { for } j=1,2,3,4 \\
\nu_{2}\left(\chi_{j}\right) & =1 \quad \text { for } j=5,6 \\
\nu_{4}\left(\chi_{j}\right) & =1 \quad \text { for } j=1,2,3,4 \\
\nu_{4}\left(\chi_{j}\right) & =2 \quad \text { for } j=5,6
\end{aligned}
$$

(4) When $H \cong H\left(-_{5}, \alpha_{1,2}^{\prime}\right)$,

$$
\begin{array}{ll}
\nu_{8}\left(\chi_{j}\right)=0 & \text { for } j=1,2,3,4 \\
\nu_{8}\left(\chi_{j}\right)=2 & \text { for } j=5,6
\end{array}
$$

(5) When $H \cong H\left(\rightarrow_{5},\left(\alpha_{1,2}^{\prime}\right)^{2}\right)$,

$$
\nu_{8}\left(\chi_{j}\right)=2 \text { for } j=1,2,3,4,5,6
$$

Proof. By Proposition 4.4, up to equivalence, there are four Hopf algebras of this type. Three of them, $H\left(\overrightarrow{-}_{5}, \alpha_{1,2}^{\prime}\right), H\left(\overrightarrow{-}_{5},\left(\alpha_{1,2}^{\prime}\right)^{2}\right)$, and $H\left(\overrightarrow{-}_{5},\left(\alpha_{1,2}^{\prime}\right)^{3}\right)$, are non-trivial.

By Proposition 4.4, $H\left(\rightarrow_{5},\left(\alpha_{1,2}^{\prime}\right)^{j}\right)$ is equivalent to $H\left(\rightarrow_{5}, \beta^{j}, \alpha_{1,2}^{j}\right)$ where

$$
\beta\left(x^{a_{1}} y^{a_{2}}\right)=i^{a_{1} a_{2}} .
$$

Define

$$
\begin{aligned}
& f: G \rightarrow G \text { via } \\
& f(x)=t \rightarrow_{5} x=y \\
& f(y)=t \rightarrow_{5} y=x
\end{aligned}
$$

Then $f\left(t \overrightarrow{ }_{5} g\right)=t \overrightarrow{ }_{5} f(g)$ and

$$
\begin{aligned}
\beta \circ f\left(x^{a_{1}} y^{a_{2}}\right) & =\beta\left(x^{a_{2}} y^{a_{1}}\right)=i^{a_{2} a_{1}} \\
\alpha_{1,2} \circ(f \times f)\left(x^{a_{1}} y^{a_{2}}, x^{b_{1}} y^{b_{2}}\right) & =\alpha_{1,2}\left(x^{a_{2}} y^{a_{1}}, x^{b_{2}} y^{b_{1}}\right)=i^{a_{2} b_{1}}
\end{aligned}
$$

and therefore

$$
\begin{aligned}
\beta \circ f & =\beta \\
\alpha_{1,2} \circ(f \times f) & =\alpha_{2,1}
\end{aligned}
$$

Define $\mu \in\left(k^{G}\right)^{\times}$via

$$
\mu\left(x^{a_{1}} y^{a_{2}}\right)=i^{a_{1} a_{2}}
$$


Then, as in part 1 of Proposition 4.1, $\alpha_{2,1}(g, h) \mu(g) \mu(h) \mu(g h)^{-1}=\alpha_{1,2}^{3}(g, h)$. Moreover

$$
\beta(g) \mu^{-1}(g) \mu^{-1}\left(t \rightarrow_{5} g\right)=\beta^{3}(g)
$$

Thus $\left[\left(\sigma^{\beta}, \tau_{2,1}\right)\right]=\left[\left(\sigma^{\beta^{3}}, \tau_{1,2}^{3}\right)\right]$ and therefore

$$
H\left(\rightarrow_{5}, \alpha_{1,2}^{\prime}\right) \cong H\left(\rightarrow_{5}, \beta, \alpha_{1,2}\right) \cong H\left(\rightarrow_{5}, \beta, \alpha_{2,1}\right) \cong H\left(\rightarrow_{5}, \beta^{3}, \alpha_{1,2}^{3}\right) \cong H\left(\rightarrow_{5},\left(\alpha_{1,2}^{\prime}\right)^{3}\right) \text {. }
$$

Let $H$ be a Hopf algebra corresponding to $\rightarrow_{5}$. Then $H$ has 8 one-dimensional characters of the type $\chi_{g} \chi_{t}^{j}$ for $j=0,1$ and $g \in G^{L}=\langle x y\rangle$ where

$$
\begin{array}{ll}
\chi_{g}\left(p_{h}\right)=\delta_{g, h} & \chi_{g}(t)=1 \\
\chi_{t}\left(p_{h}\right)=\delta_{1, h} & \chi_{g}(t)=-1
\end{array}
$$

Then

$$
\begin{aligned}
\chi_{x y}^{4} & =\chi_{t}^{2}=\chi_{1}=1 \\
\chi_{x y} \chi_{t} & =\chi_{t} \chi_{x y}
\end{aligned}
$$

Set $a=\chi_{x y}, b=\chi_{t}$. Then

$$
\mathbf{G}\left(H^{*}\right)=\langle a\rangle \times\langle b\rangle \cong \mathbb{Z}_{4} \times \mathbb{Z}_{2} .
$$

$H$ has 6 two-dimensional characters $\chi_{g, t \rightarrow_{5} g}$ for $g \in\left\{x, x^{-1}, x y^{2}, x^{-1} y^{2}, x y^{-1}, x^{2}\right\}$ where

$$
\chi_{g, t \rightarrow_{5} g}\left(p_{h}\right)=\delta_{g, h}+\delta_{t \rightarrow_{5} g, h} \quad \chi_{g, t \rightarrow_{5} g}\left(p_{h} t\right)=0 .
$$

Then

$$
\begin{aligned}
\chi_{x, y}^{2} & =\chi_{x^{-1} y^{2}, x^{2} y^{-1}}^{2}=\chi_{x y}+\chi_{x y} \chi_{t}+\chi_{x^{2}, y^{2}} \\
\chi_{x^{-1}, y^{-1}}^{2} & =\chi_{x y^{2}, x^{2} y}^{2}=\chi_{x^{-1} y^{-1}}+\chi_{x^{-1} y^{-1}} \chi_{t}+\chi_{x^{2}, y^{2}} \\
\chi_{x y^{-1}, x^{-1} y}^{2} & =\chi_{x^{2}, y^{2}}^{2}=1+\chi_{t}+\chi_{x^{2} y^{2}}+\chi_{x^{2} y^{2}} \chi_{t} \\
\nu_{2}\left(\chi_{x^{2}, y^{2}}\right) & =1 \\
\nu_{2}\left(\chi_{x y^{-1}, x^{-1} y}\right) & =\tau_{t}\left(x y^{-1}, x^{-1} y\right)=1 \\
\nu_{2}\left(\chi_{g, t \rightarrow_{5} g}\right) & =0 \text { for } g \in\left\{x, x^{-1}, x y^{2}, x^{-1} y^{2}\right\}
\end{aligned}
$$

We will now compute 4-th and 8-th Frobenius-Schur indicators for the two-dimensional characters. As in [KMM, Section 4], $\Lambda=\frac{1}{2}\left(p_{1}+p_{1} t\right)$ is the integral of $H$ such that $\varepsilon(\Lambda)=1$. Then, since $\tau_{t}=\alpha_{1,2}^{\prime}$ or $\left(\alpha_{1,2}^{\prime}\right)^{2}, h\left(t \overrightarrow{-}_{5} h\right)=(x y)^{j}$, and $h^{4}=1$ for all $h \in G$,

$$
\begin{aligned}
\Delta(\Lambda) & =\frac{1}{2}\left(\sum_{h_{1} h_{2}=1} p_{h_{1}} \otimes p_{h_{2}}+\sum_{h_{1} h_{2}=1} \tau_{t}\left(h_{1}, h_{2}\right) p_{h_{1}} t \otimes p_{h_{2}} t\right) \\
\Delta_{4}(\Lambda) & =\frac{1}{2}\left(\sum_{h_{1} h_{2} h_{3} h_{4}=1} p_{h_{1}} \otimes p_{h_{2}} \otimes p_{h_{3}} \otimes p_{h_{4}}\right.
\end{aligned}
$$


ON SEMISIMPLE HOPF ALGEBRAS OF DIMENSION $2^{m}$, II.

$$
\begin{aligned}
& \left.+\sum_{h_{1} h_{2} h_{3} h_{4}=1} \tau_{t}\left(h_{1} h_{2}, h_{3} h_{4}\right) \tau_{t}\left(h_{1}, h_{2}\right) \tau_{t}\left(h_{3}, h_{4}\right) p_{h_{1}} t \otimes p_{h_{2}} t \otimes p_{h_{3}} t \otimes p_{h_{4}} t\right) \\
\Lambda^{[4]}= & \frac{1}{2}\left(\sum_{h^{4}=1} p_{h}+\sum_{\left(h\left(t \rightarrow_{5} h\right)\right)^{2}=1} \tau_{t}\left(h\left(t \rightarrow_{5} h\right), h\left(t \rightarrow_{5} h\right)\right)\left(\tau_{t}\left(h, t \rightarrow_{5} h\right)\right)^{2} p_{h}\right) \\
= & \frac{1}{2}\left(1+\sum_{h \in\langle x y\rangle \times\left\langle x^{2}\right\rangle}\left(\tau_{t}\left(h, t \rightarrow_{5} h\right)\right)^{2} p_{h}\right) \\
\Delta_{8}(\Lambda)= & \frac{1}{2}\left(\sum_{h_{1} \cdots h_{8}=1} p_{h_{1}} \otimes \cdots \otimes p_{h_{8}}+\sum_{h_{1} \cdots h_{8}=1} \tau_{t}\left(h_{1} h_{2} h_{3} h_{4}, h_{5} h_{6} h_{7} h_{8}\right) \tau_{t}\left(h_{1} h_{2}, h_{3} h_{4}\right) \tau_{t}\left(h_{5} h_{6}, h_{7} h_{8}\right)\right. \\
\left.\Lambda_{t}\left(h_{1}, h_{2}\right) \tau_{t}\left(h_{3}, h_{4}\right) \tau_{t}\left(h_{5}, h_{6}\right) \tau_{t}\left(h_{7}, h_{8}\right) p_{h_{1}} t \otimes \cdots \otimes p_{h_{8}} t\right) & \frac{1}{2}\left(\sum_{h^{8}=1} p_{h}+\sum_{\left(h\left(t \rightarrow_{5} h\right)\right)^{4}=1} \tau_{t}\left(h\left(t \rightarrow_{5} h\right)^{2}, h\left(t \rightarrow_{5} h\right)^{2}\right)\left(\tau_{t}\left(h\left(t \rightarrow_{5} h\right), h\left(t \rightarrow_{5} h\right)\right)\right)^{2}\right. \\
& \left.\cdot\left(\tau_{t}\left(h, t \rightarrow_{5} h\right)\right)^{4} p_{h}\right)=\frac{1}{2}\left(1+\sum_{h \in G}\left(\tau_{t}\left(h, t \rightarrow_{5} h\right)\right)^{4} p_{h}\right)
\end{aligned}
$$

Then

$$
\begin{aligned}
\nu_{4}\left(\chi_{g, t-_{5} g}\right) & =1 \quad \text { for } g \in\left\{x, x^{-1}, x y^{2}, x^{-1} y^{2}\right\} \\
\nu_{4}\left(\chi_{x y^{-1}, x^{-1} y}\right) & =\frac{1}{2}\left(2+\left(\tau_{t}\left(x y^{3}, x^{3} y\right)\right)^{2}+\left(\tau_{t}\left(x^{3} y, x y^{3}\right)\right)^{2}\right)=\frac{1}{2}(2+1+1)=2 \\
\nu_{4}\left(\chi_{x^{2}, y^{2}}\right) & =\frac{1}{2}\left(2+\left(\tau_{t}\left(x^{2}, y^{2}\right)\right)^{2}+\left(\tau_{t}\left(y^{2}, x^{2}\right)\right)^{2}\right)=\frac{1}{2}(2+1+1)=2
\end{aligned}
$$

If $H \cong H\left(\rightarrow_{5},\left(\alpha_{1,2}^{\prime}\right)^{2}\right)$ then $\Lambda^{[8]}=1$ and

$$
\nu_{8}\left(\chi_{g, t \rightarrow_{5} g}\right)=2 \quad \text { for all } g \in\left\{x, x^{-1}, x y^{2}, x^{-1} y^{2}, x y^{-1}, x^{2}\right\}
$$

If $H \cong H\left(\rightarrow_{5}, \alpha_{1,2}^{\prime}\right)$ then

$$
\begin{aligned}
\nu_{8}\left(\chi_{x y^{-1}, x^{-1} y}\right) & =\nu_{8}\left(\chi_{x^{2}, y^{2}}\right)=\frac{1}{2}(2+1+1)=2 \\
\nu_{8}\left(\chi_{g, t-_{5} g}\right) & =\frac{1}{2}(2+(-1)+(-1))=0 \quad \text { for } g \in\left\{x, x^{-1}, x y^{2}, x^{-1} y^{2}\right\} .
\end{aligned}
$$

Moreover, note that $\exp \left(H\left(\overrightarrow{-}_{5},\left(\alpha_{1,2}^{\prime}\right)^{2}\right)\right)=8$ and $\exp \left(H\left(\overrightarrow{-}_{5}, \alpha_{1,2}^{\prime}\right)\right)=16$. 
Proposition 5.6. Assume that

$$
t \rightarrow_{6} x=x y^{2}, \quad t \rightarrow_{6} y=x^{2} y^{-1} .
$$

Then $H \cong H\left(\rightarrow_{6}, \alpha_{1,1} \alpha_{1,2} \alpha_{2,2}\right)$ or $H\left(\rightarrow_{6}, \alpha_{1,1}^{2} \alpha_{1,2}^{2} \alpha_{2,2}^{2}\right)$. Moreover

(1) $\mathbf{G}(H) \cap Z(H)=\hat{G}^{L}=\left\langle\beta_{1}^{2}\right\rangle \times\left\langle\beta_{2}^{2}\right\rangle \cong \mathbb{Z}_{2} \times \mathbb{Z}_{2}$.

(2) $\mathbf{G}\left(H^{*}\right)=\langle a\rangle \times\langle b\rangle \times\langle c\rangle \cong \mathbb{Z}_{2} \times \mathbb{Z}_{2} \times \mathbb{Z}_{2}$.

(3) $H$ has 6 two-dimensional representations $\pi_{1}, \ldots, \pi_{6}$ with characters $\chi_{1}, \ldots, \chi_{6}$ such that

$$
\begin{aligned}
\pi_{1}^{2} & =\pi_{2}^{2}=a+a c+a b+a b c \\
\pi_{3}^{2} & =\pi_{4}^{2}=a+a c+b+b c \\
\pi_{5}^{2} & =\pi_{6}^{2}=a b+a b c+b+b c \\
\nu_{2}\left(\chi_{j}\right) & =0 \quad \text { for } j=1,2,3,4,5,6
\end{aligned}
$$

(4) When $H \cong H\left(\rightarrow_{6}, \alpha_{1,1} \alpha_{1,2} \alpha_{2,2}\right)$,

$$
\nu_{4}\left(\chi_{j}\right)=0 \quad \text { for } j=1,2,3,4,5,6
$$

(5) When $H \cong H\left({ }_{6}, \alpha_{1,1}^{2} \alpha_{1,2}^{2} \alpha_{2,2}^{2}\right)$,

$$
\nu_{4}\left(\chi_{j}\right)=2 \text { for } j=1,2,3,4,5,6
$$

Proof. By Proposition 4.18, up to equivalence, there are four Hopf algebras of this type. Three of them, $H\left(\rightarrow_{6}, \alpha_{1,1} \alpha_{1,2} \alpha_{2,2}\right), H\left(\rightarrow_{6}, \alpha_{1,1}^{2} \alpha_{1,2}^{2} \alpha_{2,2}^{2}\right)$, and $H\left(\rightarrow_{6}, \alpha_{1,1}^{3} \alpha_{1,2}^{3} \alpha_{2,2}^{3}\right)$, are non-trivial.

Define

$$
\begin{aligned}
& f: G \rightarrow G \text { via } \\
& f(x)=t \rightarrow_{6} x=x y^{2} \\
& f(y)=t \rightarrow_{6} y=x^{2} y^{-1}
\end{aligned}
$$

Then $f\left(t \rightarrow_{6} g\right)=t \rightarrow_{6} f(g)$ and

$$
\begin{aligned}
\alpha_{1,1} \alpha_{1,2} \alpha_{2,2} \circ(f \times f)\left(x^{a_{1}} y^{a_{2}}, x^{b_{1}} y^{b_{2}}\right) & =\alpha_{1,1} \alpha_{1,2} \alpha_{2,2}\left(x^{a_{1}+2 a_{2}} y^{2 a_{1}-a_{2}}, x^{b_{1}+2 b_{2}} y^{2 b_{1}-b_{2}}\right) \\
& =i^{a_{1} b_{1}}(-1)^{a_{1} b_{2}+a_{2} b_{1}} i^{-a_{1} b_{2}}(-1)^{a_{1} b_{1}+a_{2} b_{2}} i^{a_{2} b_{2}}(-1)^{a_{1} b_{2}+a_{2} b_{1}} \\
& =i^{-a_{1} b_{1}-a_{1} b_{2}-a_{2} b_{2}}
\end{aligned}
$$

and therefore

$$
\alpha_{1,1} \alpha_{1,2} \alpha_{2,2} \circ(f \times f)=\alpha_{1,1}^{3} \alpha_{1,2}^{3} \alpha_{2,2}^{3}
$$

Thus $H\left(\rightarrow_{6}, \alpha_{1,1} \alpha_{1,2} \alpha_{2,2}\right) \cong H\left(\rightarrow_{6} \alpha_{1,1}^{3} \alpha_{1,2}^{3} \alpha_{2,2}^{3}\right)$.

Let $H$ be a Hopf algebra corresponding to $\rightarrow_{6}$. Then $H$ has 8 one-dimensional characters of the type $\chi_{g} \chi_{t}^{j}$ for $j=0,1$ and $g \in G^{L}=\left\langle x^{2}\right\rangle \times\left\langle y^{2}\right\rangle$ where

$$
\begin{array}{ll}
\chi_{g}\left(p_{h}\right)=\delta_{g, h} & \chi_{g}(t)=1 \\
\chi_{t}\left(p_{h}\right)=\delta_{1, h} & \chi_{g}(t)=-1
\end{array}
$$


For each one-dimensional character $\chi, \chi^{2}=1$. Set $a=\chi_{x^{2}}, b=\chi_{y^{2}}$ and $c=\chi_{t}$. Then

$$
\mathbf{G}\left(H^{*}\right)=\langle a\rangle \times\langle b\rangle \times\langle c\rangle \cong \mathbb{Z}_{2} \times \mathbb{Z}_{2} \times \mathbb{Z}_{2} .
$$

$H$ has 6 two-dimensional characters $\chi_{g, t \rightarrow} g$ for $g \in\left\{x, x^{-1}, y, y^{-1}, x y, x y^{-1}\right\}$ where

$$
\chi_{g, t \rightarrow_{6} g}\left(p_{h}\right)=\delta_{g, h}+\delta_{t \rightarrow} g, h \quad \chi_{g, t \rightarrow} g\left(p_{h} t\right)=0 .
$$

Then

$$
\begin{aligned}
& \chi_{x, x y^{2}}^{2}=\chi_{x^{-1}, x^{-1} y^{2}}^{2}=\chi_{x^{2}}+\chi_{x^{2}} \chi_{t}+\chi_{x^{2} y^{2}}+\chi_{x^{2} y^{2}} \chi_{t} \\
& \chi_{y, x^{2} y^{-1}}^{2}=\chi_{y^{-1}, x^{2} y}^{2}=\chi_{x^{2}}+\chi_{x^{2}} \chi_{t}+\chi_{y^{2}}+\chi_{y^{2}} \chi_{t} \\
& \chi_{x y, x^{-1} y}^{2}=\chi_{x y^{-1}, x^{-1} y^{-1}}^{2}=\chi_{x^{2} y^{2}}+\chi_{x^{2} y^{2}} \chi_{t}+\chi_{y^{2}}+\chi_{y^{2}} \chi_{t} \\
& \nu_{2}\left(\chi_{g, t \rightarrow_{6} g}\right)=0 \quad \text { for all } g \in\left\{x, x^{-1}, y, y^{1}, x y, x y^{-1}\right\}
\end{aligned}
$$

We will now compute 4-th Frobenius-Schur indicators for the two-dimensional characters. Recall that $\Lambda=\frac{1}{2}\left(p_{1}+p_{1} t\right)$ is the integral of $H$ such that $\varepsilon(\Lambda)=1$. Then, since $\tau_{t}=\alpha_{1,1} \alpha_{1,2} \alpha_{2,2}$ or $\alpha_{1,1}^{2} \alpha_{1,2}^{2} \alpha_{2,2}^{2}, h\left(t \rightarrow_{6} h\right)=x^{2 j} y^{2 k}$, and $h^{4}=1$ for all $h \in G$,

$$
\begin{aligned}
\Delta(\Lambda)= & \frac{1}{2}\left(\sum_{h_{1} h_{2}=1} p_{h_{1}} \otimes p_{h_{2}}+\sum_{h_{1} h_{2}=1} \tau_{t}\left(h_{1}, h_{2}\right) p_{h_{1}} t \otimes p_{h_{2}} t\right) \\
\Delta_{4}(\Lambda)= & \frac{1}{2}\left(\sum_{h_{1} h_{2} h_{3} h_{4}=1} p_{h_{1}} \otimes p_{h_{2}} \otimes p_{h_{3}} \otimes p_{h_{4}}\right. \\
& \left.+\sum_{h_{1} h_{2} h_{3} h_{4}=1} \tau_{t}\left(h_{1} h_{2}, h_{3} h_{4}\right) \tau_{t}\left(h_{1}, h_{2}\right) \tau_{t}\left(h_{3}, h_{4}\right) p_{h_{1}} t \otimes p_{h_{2}} t \otimes p_{h_{3}} t \otimes p_{h_{4}} t\right) \\
\Lambda^{[4]}= & \frac{1}{2}\left(\sum_{h^{4}=1} p_{h}+\sum_{\left(h\left(t \rightarrow{ }_{6} h\right)\right)^{2}=1} \tau_{t}\left(h\left(t \rightarrow_{6} h\right), h\left(t \rightarrow_{6} h\right)\right)\left(\tau_{t}\left(h, t \rightarrow_{6} h\right)\right)^{2} p_{h}\right) \\
= & \frac{1}{2}\left(1+\sum_{h \in G}\left(\tau_{t}\left(h, t \rightarrow_{6} h\right)\right)^{2} p_{h}\right)
\end{aligned}
$$

If $H \cong H\left(\rightarrow_{6}, \alpha_{1,1}^{2} \alpha_{1,2}^{2} \alpha_{2,2}^{2}\right)$ then $\Lambda^{[4]}=1$ and

$$
\nu_{4}\left(\chi_{g, t \rightarrow} g\right)=2 \quad \text { for all } g \in\left\{x, x^{-1}, y, y^{-1}, x y, x y^{-1}\right\} .
$$

If $H \cong H\left(\rightarrow_{6}, \alpha_{1,1} \alpha_{1,2} \alpha_{2,2}\right)$ then

$$
\nu_{4}\left(\chi_{g, t \rightarrow_{6} g}\right)=\frac{1}{2}(2+(-1)+(-1))=0 \quad \text { for all } g \in\left\{x, x^{-1}, y, y^{-1}, x y, x y^{-1}\right\} .
$$

Moreover, note that $\exp \left(H\left(\rightarrow_{6}, \alpha_{1,1}^{2} \alpha_{1,2}^{2} \alpha_{2,2}^{2}\right)\right)=4$ and $\exp \left(H\left(\rightarrow_{6}, \alpha_{1,1} \alpha_{1,2} \alpha_{2,2}\right)\right)=$ 8. 
Since Hopf algebras associated with two different actions are isomorphic only if the corresponding matrices are similar, the above six propositions can be combined in the following theorem:

Theorem 5.7. There are exactly 16 nontrivial non-isomorphic semisimple Hopf algebras of dimension 32 with group of group-like elements isomorphic to $\mathbb{Z}_{4} \times \mathbb{Z}_{4}$. Moreover, all of them can be distinguished by categorical invariants, such as their Grothendieck ring structure or their (higher) Frobenius-Schur indicators. Therefore, the corresponding representation categories are also different and these Hopf algebras are not twist-equivalent to each other.

\section{REFERENCES}

[A] N. Andruskiewitsch, Notes on extensions of Hopf algebras, Canadian J. Math 48 (1996), $3-42$.

[H] I. Hofstetter, Extensions of Hopf algebras and their cohomological description, J. Algebra 164 (1994), 264-298.

[Ka] G. Karpilovsky, The Schur multiplier. London Mathematical Society Monographs. New Series, 2. The Clarendon Press, Oxford University Press, New York, 1987.

[K1] Y. Kashina, Classification of semisimple Hopf algebras of dimension 16, J. Algebra, 232 (2000), no. 2, 617-663.

[K2] Y. Kashina, On semisimple Hopf algebras of dimension $2^{m}$, Algebr. Represent. Theory, 6 (2003), no. 4, 393-425.

[K3] Y. Kashina, Groups of grouplike elements of a semisimple Hopf algebra and its dual, Hopf algebras and generalizations, 53-62, Contemp. Math., 441, Amer. Math. Soc., Providence, RI, 2007.

[KMM] Y. Kashina, G. Mason, and S. Montgomery, Computing the Frobenius-Schur indicator for abelian extensions of Hopf algebras. J. Algebra 251 (2002), no. 2, 888-913.

[Kr] L. Krop, On classification of finite-dimensional semisimple Hopf algebras. ar Xiv:1503.06198.

[LM] V. Linchenko and S. Montgomery, A Frobenius-Schur theorem for Hopf algebras, Algebras and Representation Theory, 3 (2000), no. 4, 347-355.

[Mac] S. Mac Lane, Homology. Reprint of the 1975 edition. Classics in Mathematics. SpringerVerlag, Berlin, 1995.

[Mas1] M. Mastnak, Hopf algebra extensions arising from semi-direct products of groups. J. Algebra 251 (2002), no. 1, 413-434.

[Mas2] M. Mastnak, On Hopf algebra extensions arising from semi-direct products of groups. Groups, rings, Lie and Hopf algebras (St. John's, NF, 2001), 129-139, Math. Appl., 555, Kluwer Acad. Publ., Dordrecht, 2003.

[Ma1] A. Masuoka, Selfdual Hopf algebras of dimension $p^{3}$ obtained by extension. J. Algebra 178 (1995), no. 3, 791-806.

[Ma2] A. Masuoka, The $p^{n}$ theorem for semisimple Hopf algebras. Proc. Amer. Math. Soc. 124 (1996), no. 3, 735-737.

[Ma3] A. Masuoka, Some further classification results on semisimple Hopf algebras. Comm. Algebra 24 (1996), no. 1, 307-329.

[Ma4] A. Masuoka, Calculations of some groups of Hopf algebra extensions, J. Algebra 191 (1997), no. 2, 568-588; Corrigendum, J. Algebra 191 (1997), no. 2, 656

[Ma5] A. Masuoka, Hopf algebra extensions and cohomology, New directions in Hopf algebras, 167-209, Math. Sci. Res. Inst. Publ., 43, Cambridge Univ. Press, Cambridge, 2002. 
[M] S. Montgomery, Hopf Algebras and Their Actions on Rings, CBMS Lectures vol. 82, AMS, Providence, RI, 1993.

[MW] S. Montgomery and S. J. Witherspoon, Irreducible representations of crossed products, J. Pure Appl. Algebra 129 (1998), no. 3, 315-326.

[Na1] S. Natale, On semisimple Hopf algebras of dimension $p q^{2}$, J. Algebra 221 (1999), 242-278.

[Na2] S. Natale, On semisimple Hopf algebras of dimension $p q^{2}$. II, Algebr. Represent. Theory 4 (2001), no. 3, 277-291.

[Na3] S. Natale, On semisimple Hopf algebras of dimension pqr, Algebr. Represent. Theory 7 (2004), no. 2, 173-188.

[Na4] S. Natale, Semisolvability of semisimple Hopf algebras of low dimension, Memoirs Amer. Math. Soc, Vol. 186, No. 874, Am. Math. Soc., Providence, 2007.

[Na5] S. Natale, Hopf algebra extensions of group algebras and Tambara-Yamagami categories. Algebr. Represent. Theory 13 (2010), no. 6, 673-691.

[Na6] S. Natale, Semisimple Hopf algebras of dimension 60. J. Algebra 324 (2010), no. 11, 30173034 .

[NR] W. Nichols and M. B. Richmond, The Grothendieck group of a Hopf algebra, J. Pure and Appl. Algebra 106 (1996), 297-306.

[Se] J.-P. Serre, Groups de Grothendieck des schemas en groups réductifs déployés, Publ. Math I.H.E.S., 34 (1968), 37-52.

[W] S. Witherspoon, Clifford correspondence for finite-dimensional Hopf algebras, J. Algebra 218 (1999), 608-620.

Department of Mathematical Sciences, DePaul University, Chicago, IL, 60614

E-mail address: ykashina@depaul.edu 